\title{
Electrical conductivity tensor of dense plasma in magnetic fields
}

\section{Arus Harutyunyan*}

Institute for Theoretical Physics, J.-W. Goethe University, Frankfurt am Main

aruseth.physik.uni-frankfurt.de

\section{Armen Sedrakian}

Institute for Theoretical Physics, J.-W. Goethe University, Frankfurt am Main

sedrakianeth.physik.uni-frankfurt.de

\begin{abstract}
Electrical conductivity of finite-temperature plasma in neutron star crusts is studied for applications in magneto-hydrodynamical description of compact stars. We solve the Boltzmann kinetic equation in relaxation time approximation taking into account the anisotropy of transport due to the magnetic field, the effects of dynamical screening in the scattering matrix element and correlations among the nuclei. We show that conductivity has a minimum at a non-zero temperature, a low-temperature decrease and a power-law increase with increasing temperature. Selected numerical results are shown for matter composed of carbon, iron, and heavier nuclei present in the outer crusts of neutron star.
\end{abstract}

The Modern Physics of Compact Stars 2015

30 September 2015 - 3 October 2015

Yerevan, Armenia

\footnotetext{
* Speaker.
} 


\section{Introduction}

Magneto-hydrodynamics (MHD) forms the basis of large-scale description of physics of dense plasma in compact stars. A key quantity in the dissipative formulations of MHD is the conductivity of matter. It determines, for example, the dissipation of currents and therefore the decay of magnetic fields, the dispersion of plasma waves, etc. In turn, magnetic field decay affects the rotational and thermal evolutions of neutron stars and consequently a broad array of their observational manifestations.

Transport in compact star plasma was studied traditionally in the cold (essentially zero-temperature) and dense regime where the constituents form degenerate quantum liquids. This regime is relevant for mature isolated or accreting neutron stars as well as interiors of white dwarfs. The dilute and warm (non-zero temperature) regime is of interest in the context of transient, shortlived states of neutron stars, such as proto-neutron stars newly born in supernova explosions or hypermassive remnants formed in the aftermath of neutron star binary mergers.

We start this article with an overview of the transport calculations of electrical conductivity of compact star matter in the density regime corresponding to their outer crusts $\left(\rho \leq 10^{11} \mathrm{~g} \mathrm{~cm}^{-3}\right)$. Then we go on to describe our recent effort to calculate the electrical conductivity of non-zero temperature crustal plasma. We focus on sufficiently high temperatures where nuclei form a liquid coexisting with electronic background of arbitrary degeneracy. We close this review with a summary and outlook. Below we use the natural (Gaussian) units with $\hbar=c=k_{B}=k_{e}=1, e=\sqrt{\alpha}$, $\alpha=1 / 137$ and the metric signature $(1,-1,-1,-1)$.

\section{Overview}

At densities relevant to interiors of white dwarfs and neutron star crusts the electron-ion system is in a plasma state - the ions are fully ionized while free electrons are the most mobile carriers of charge. By charge conservation electron density is related to the ion charge $Z$ by $n_{e}=Z n_{i}$, where $n_{i}$ is the number density of nuclei. Electrons to a good accuracy form non-interacting gas which becomes degenerate below the Fermi temperature $T_{F}=\varepsilon_{F}-m=\left(p_{F}^{2}+m^{2}\right)^{1 / 2}-m$, where the electron Fermi momentum is given by $p_{F}=\left(3 \pi^{2} n_{e}\right)^{1 / 3}$ and $m$ is the electron mass. The state of ions (mass number $A$ and charge $Z$ ) is controlled by the value of the Coulomb plasma parameter $\Gamma$

$$
\Gamma=\frac{e^{2} Z^{2}}{T a_{i}} \approx 22.73 \frac{Z^{2}}{T_{6}}\left(\frac{\rho_{6}}{A}\right)^{1 / 3},
$$

where $e$ is the elementary charge, $T$ is the temperature, $a_{i}=\left(4 \pi n_{i} / 3\right)^{-1 / 3}$ is the radius of the spherical volume per ion, $T_{6}$ is the temperature in units $10^{6} \mathrm{~K}$ and $\rho_{6}$ is the density in units of $10^{6}$ $\mathrm{g} \mathrm{cm}^{-3}$. If $\Gamma \ll 1$ or, equivalently $T \gg T_{\mathrm{C}} \equiv Z^{2} e^{2} / a_{i}$, ions form weakly coupled Boltzmann gas. In the regime $\Gamma \geq 1$ ions are strongly coupled and form a liquid for low values of $\Gamma \leq \Gamma_{m} \simeq 160$ and a lattice for $\Gamma>\Gamma_{m}$. The melting temperature of the lattice associated with $\Gamma_{m}$ is defined as $T_{m}=(Z e)^{2} / \Gamma_{m} a_{i}$. For temperatures below the ion plasma temperature

$$
T_{p}=\left(\frac{4 \pi Z^{2} e^{2} n_{i}}{M}\right)^{1 / 2}
$$




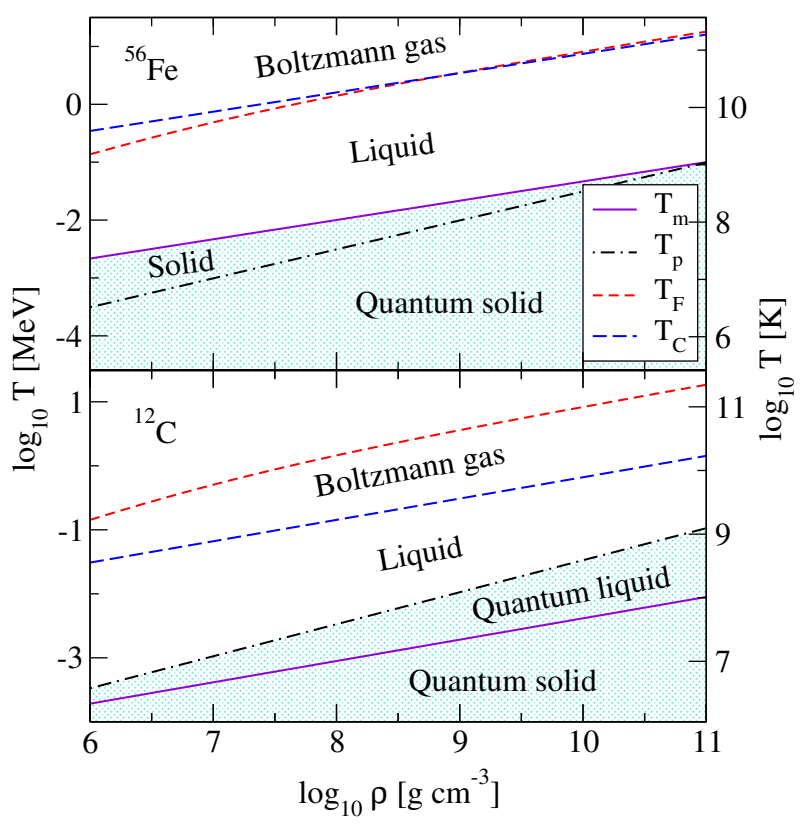

Figure 1: The temperature-density phase diagram of dense plasma composed of iron ${ }^{56} \mathrm{Fe}$ (upper panel) and carbon ${ }^{12} \mathrm{C}$ (lower panel). The electron gas degeneracy sets in below the Fermi temperature $T_{F}$ (short dashed lines). The ionic component solidifies below the melting temperature $T_{m}$ (solid lines), while quantum effects become important below the plasma temperature (dash-dotted lines). For temperatures above $T_{\mathrm{C}}$ (long dashed lines) the ionic component forms a Boltzmann gas. Note that for ${ }^{12} \mathrm{C}$ the quantum effects become important in the portion of the phase diagram lying between the lines $T_{p}(\rho)$ and $T_{m}(\rho)$. The present study does not cover the shaded portion of the phase diagram.

where $M$ is the ion mass, the quantization of oscillations of the lattice becomes important. Figure 1 shows the temperature-density phase diagram of the crustal material in the cases where it is composed of iron ${ }^{56} \mathrm{Fe}$ (left panel) or carbon ${ }^{12} \mathrm{C}$ (right panel).

While the structure of the phase diagrams for ${ }^{56} \mathrm{Fe}$ and ${ }^{12} \mathrm{C}$ are similar there is an important difference as well: as the temperature is lowered the quantum effects become important for carbon prior to solidification, whereas iron solidifies close to the temperature where ionic quantum effects become important. Except of hydrogen and perhaps helium which may not solidify because of quantum zero point motions all heavier elements $Z>2$ solidify at low enough temperature. The phase diagram in the case of density dependent composition is shown in Fig. 2.

The earliest studies of transport in dense matter go back to the work by Mestel, Hoyle [1] and Lee [2] in 1950s, who obtained the "conductive opacity", or equivalently the thermal conductivity of the electron-ion plasma in non-relativistic electron regime in the context of radiative and thermal transport in white dwarfs. Above densities of the order of $10^{6} \mathrm{~g} \mathrm{~cm}^{-3}$ electrons are relativistic. Following the initial qualitative estimates of the conductivity of highly compressed matter by Abrikosov in 1963 [3] more detailed calculations were carried out in the 1970s by many authors. In particular the transport in neutron star crusts in the relativistic electron regime was studied in much detail by Flowers and Itoh [4] both in the solid and in the liquid regime using a variational 


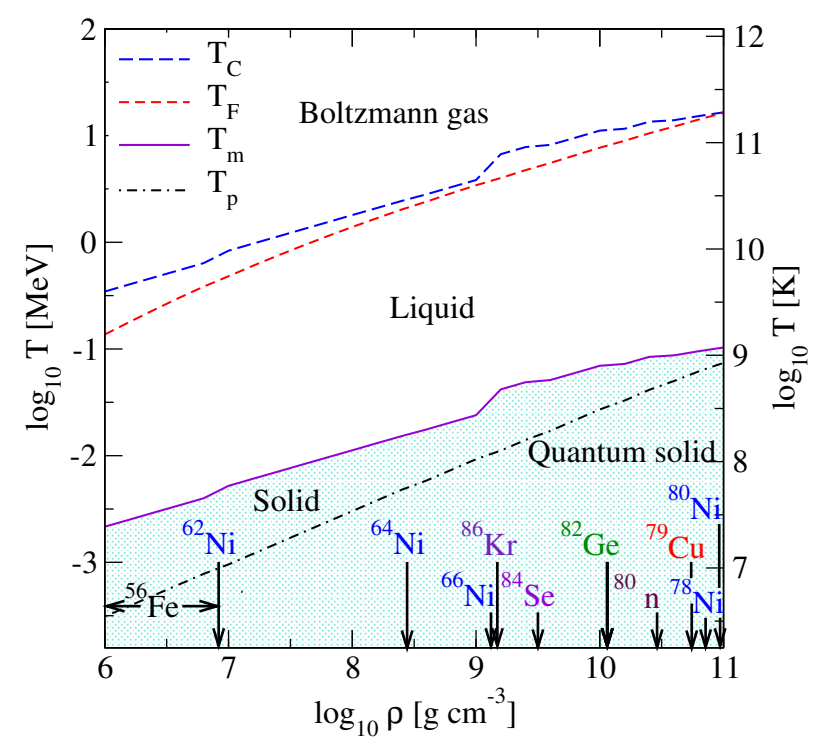

Figure 2: Same as in Fig. 1 but for density dependent composition; for details and references see the discussion in Ref. [12].

method. They were able to cover a broad range of densities and low-temperature regime including multiple channels of scattering, relatively accurate description of collective modes (phonons) which contribute to the transport coefficients in the solid phase. Their discussion also extended to the neutron drip region where free neutrons contribute to the thermal conductivity and shear viscosity of matter. A critical analysis of the numerical values for the transport coefficients found by different authors was given by Yakovlev and Urpin [5], who also provided useful and simple approximations for the transport coefficients in the degenerate electron regime in terms of the Coulomb logarithm. Nandkumar and Pethick [6] studied the temperature regime above the melting temperature, i.e., where ions form a liquid, showing that the screening of electron-ion interactions can lead to substantial corrections in this case. These calculations agree with those of Itoh et al. [7] who also provide useful fitting formulae for the transport coefficient. Subsequent refinements of the results quoted above included, among other things, multi-phonon process and Debye-Waller factor [8] in the solid phase and improved correlation functions in the liquid phase [9]. The implementation of the transport coefficients of dense matter in the dissipative MHD equations in the case of cold neutron crust plasma in the presence of magnetic fields were discussed by a number of authors $[10,11]$. We do not discuss here the physics in ultra-strong fields and confine our attention to non-quantizing fields, i.e., fields below the critical field $B \simeq 10^{14} \mathrm{G}$ above which the Landau quantization of electron trajectories becomes important.

\section{Formalism}

The Boltzmann equation for electron distribution function is given by

$$
\frac{\partial f}{\partial t}+\boldsymbol{V} \frac{\partial f}{\partial \boldsymbol{r}}-e(\boldsymbol{E}+\boldsymbol{V} \times \boldsymbol{H}) \frac{\partial f}{\partial \boldsymbol{p}}=I[f]
$$


where $\boldsymbol{V}$ is the electron velocity, $\boldsymbol{E}$ and $\boldsymbol{H}$ are the electric and magnetic fields and $I$ is the collision intergal. We are interested in the regime where the collision integral describes electron-ion scattering and, therefore, has the form

$$
I[f]=-(2 \pi)^{4} \sum_{234}\left|M_{12 \rightarrow 34}\right|^{2} \delta^{(4)}\left(p+p_{2}-p_{3}-p_{4}\right)\left[f\left(1-f_{3}\right) g_{2}-f_{3}(1-f) g_{4}\right],
$$

where $p_{i}$ are four-momenta of particles, $g$ is the equilibrium distribution function of ions, which to a good accuracy can be described by the Maxwell-Boltzmann distribution with energy spectrum $\varepsilon=p^{2} / 2 M$, where $M$ is the ion mass. The sum Eq. (3.2) stands symbolically for the integrals over the phase-space of scattering particles, $M_{12 \rightarrow 34}$ is the transition matrix element for scattering of relativistic electrons off correlated ions and is given by

$$
M_{12 \rightarrow 34}=\frac{J_{0} J_{0}^{\prime}}{q^{2}+\Pi_{l}(\omega, \boldsymbol{q})}-\frac{\boldsymbol{J}_{t} \boldsymbol{J}_{t}^{\prime}}{q^{2}-\omega^{2}+\Pi_{t}(\omega, \boldsymbol{q})},
$$

where the electron and ion four-currents are given, respectively,

$$
J^{\mu}=-e^{*} \bar{u}^{s_{3}}\left(p_{3}\right) \gamma^{\mu} u^{s}(p), \quad J^{\prime \mu}=Z e^{*} V^{\prime \mu}=Z e^{*}\left(1, \boldsymbol{p}^{\prime} / M\right),
$$

$e^{*}=\sqrt{4 \pi} e$, and $J_{t}, J_{t}^{\prime}$ are the components of the currents transversal to the moment transfer $\boldsymbol{q}$, $\Pi_{l}(\omega, \boldsymbol{q})$ and $\Pi_{t}(\omega, \boldsymbol{q})$ are the longitudinal and transverse components of the polarization tensor, which describe respectively, the (irreducible) self-energies of longitudinal and transverse photons in the medium (plasma). The form of the matrix element (3.3) includes thus the dynamical screening of the electron-ion interaction due to the exchange of transverse photons. Such separation has been employed in the treatment of transport in unpaired [13] and superconducting quark matter [14] and we adopt an analogous approach here. We linearize the Boltzmann equation (3.1) by writing

$$
f=f^{0}+\delta f, \quad \delta f=-\Phi \frac{\partial f^{0}}{\partial \varepsilon}
$$

where $f^{0}$ is the equilibrium Fermi-Dirac distribution function, $\delta f \ll f^{0}$, and $\Phi$ is the perturbation. The electric field appears in the drift term of linearized Boltzmann equation at $O(1)$ in perturbation, whereas the term involving magnetic field at order $O(\Phi)$, because $[\boldsymbol{V} \times \boldsymbol{H}]\left(\partial f^{0} / \partial \boldsymbol{p}\right) \propto[\boldsymbol{V} \times \boldsymbol{H}] \boldsymbol{V}=$ 0 . We next specify the form of the function $\Phi$ in the case of conduction as $\Phi=\boldsymbol{p} \cdot \boldsymbol{\Xi}(\varepsilon)$, which after substitution in the linearized Boltzmann equation gives

$$
e \boldsymbol{V} \cdot[\boldsymbol{E}+(\boldsymbol{\Xi} \times \boldsymbol{H})]=-\boldsymbol{\Xi} \cdot \boldsymbol{p} \tau^{-1}(\varepsilon),
$$

where the relaxation time, which depends on electron energy $\varepsilon$, is defined by

$$
\tau^{-1}(\varepsilon)=(2 \pi)^{-5} \int d \omega d \boldsymbol{q} \int d \boldsymbol{p}_{2}\left|M_{12 \rightarrow 34}\right|^{2} \frac{\boldsymbol{q} \cdot \boldsymbol{p}}{p^{2}} \delta\left(\varepsilon-\varepsilon_{3}-\omega\right) \delta\left(\varepsilon_{2}-\varepsilon_{4}+\omega\right) g_{2} \frac{1-f_{3}^{0}}{1-f^{0}}
$$

(Here and below the indices 2 and 4 are reserved for ions, the index 3 corresponds to the outgoing electron). In transforming the linearized collision integral we introduced a dummy integration over energy and momentum transfers, i.e., $\omega=\varepsilon-\varepsilon_{3}$ and $\boldsymbol{q}=\boldsymbol{p}-\boldsymbol{p}_{3}$. It remains to express the vector 
$\Xi$ describing the perturbation in terms of physical fields; its most general decomposition is given by

$$
\boldsymbol{\Xi}=\alpha \boldsymbol{e}+\beta \boldsymbol{h}+\gamma[\boldsymbol{e} \times \boldsymbol{h}],
$$

where $\boldsymbol{h} \equiv \boldsymbol{H} / H$ and $\boldsymbol{e} \equiv \boldsymbol{E} / E$ and the coefficients $\alpha, \beta, \gamma$ are functions of the electron energy. Substituting Eq. (3.8) in Eq. (3.6) one finds that $\alpha=-e E \tau / \varepsilon\left(1+\omega_{c}^{2} \tau^{2}\right), \beta / \alpha=\left(\omega_{c} \tau\right)^{2}(\boldsymbol{e} \cdot \boldsymbol{h})$ and $\gamma / \alpha=-\omega_{c} \tau$, where $\omega_{c}=e H \varepsilon^{-1}$ is the cyclotron frequency. As a result, the most general form of the perturbation is given by

$$
\Phi=-\frac{e \tau}{1+\left(\omega_{c} \tau\right)^{2}} V_{i}\left[\delta_{i j}-\omega_{c} \tau \varepsilon_{i j k} h_{k}+\left(\omega_{c} \tau\right)^{2} h_{i} h_{j}\right] E_{j}
$$

Using the standard expression for the electrical current in terms of the perturbation $\Phi$ we arrive at the conductivity tensor $\sigma_{i j}=\delta_{i j} \sigma_{0}-\varepsilon_{i j m} h_{m} \sigma_{1}+h_{i} h_{j} \sigma_{2}$, where the components of the tensor are defined as

$$
\sigma_{n}=\frac{e^{2}}{3 \pi^{2} T} \int_{m}^{\infty} d \varepsilon \frac{p^{3}}{\varepsilon} \frac{\tau\left(\omega_{c} \tau\right)^{n}}{1+\left(\omega_{c} \tau\right)^{2}} f^{0}\left(1-f^{0}\right), \quad n=0,1,2
$$

where $T$ is the temperature and the lower bound of the integral is given by the mass of the electron, which vanishes in the ultra-relativistic limit. The conductivity tensor has a particularly simple form if the magnetic field is along the $z$-direction

$$
\hat{\sigma}=\left(\begin{array}{ccc}
\sigma_{0} & -\sigma_{1} & 0 \\
\sigma_{1} & \sigma_{0} & 0 \\
0 & 0 & \sigma
\end{array}\right) .
$$

For zero magnetic field the current is along the electric field and we find the scalar conductivity

$$
\sigma=\frac{e^{2}}{3 \pi^{2} T} \int_{m}^{\infty} d \varepsilon \frac{p^{3}}{\varepsilon} \tau f^{0}\left(1-f^{0}\right)=\sigma_{0}+\sigma_{2}
$$

Thus, the components of the conductivity tensor are fully determined if the relaxation time $\tau$ is known. We evaluate the square of the scattering matrix using the standard QFT methods and then average over the positions of correlated ions, which effectively multiplies the transition probability by the structure function of ions. After some computations we find for the relaxation time

$$
\begin{aligned}
\tau^{-1}(\varepsilon) & =\frac{\pi Z^{2} e^{4} n_{i}}{\varepsilon p^{3}} \int_{-\infty}^{\varepsilon-m} d \omega e^{-\omega / 2 T} \frac{f^{0}(\varepsilon-\omega)}{f^{0}(\varepsilon)} \int_{q_{-}}^{q_{+}} d q\left(q^{2}-\omega^{2}+2 \varepsilon \omega\right) S(q) F^{2}(q) \frac{1}{\sqrt{2 \pi} \theta} \\
& \times e^{-\omega^{2} / 2 q^{2} \theta^{2}} e^{-q^{2} / 8 M T}\left\{\frac{(2 \varepsilon-\omega)^{2}-q^{2}}{\left|q^{2}+\Pi_{l}\right|^{2}}+\theta^{2} \frac{\left(q^{2}-\omega^{2}\right)\left[(2 \varepsilon-\omega)^{2}+q^{2}\right]-4 m^{2} q^{2}}{q^{2}\left|q^{2}-\omega^{2}+\Pi_{t}\right|^{2}}\right\},
\end{aligned}
$$

where $S(q)$ is the ionic structure function, $\theta \equiv \sqrt{T / M}, q_{ \pm}=\left| \pm p+\sqrt{p^{2}-\left(2 \omega \varepsilon-\omega^{2}\right)}\right|$ and $\varepsilon=\sqrt{p^{2}+m^{2}}$ for non-interacting electrons. The contribution of longitudinal and transverse photons in (3.13) separate. The dynamical screening effects contained in the transverse contribution are parametrically suppressed by the factor $T / M$ at low temperatures and for heavy nuclei. This contribution is clearly important in the cases where electron-electron $(e-e)$ scattering contributes to 


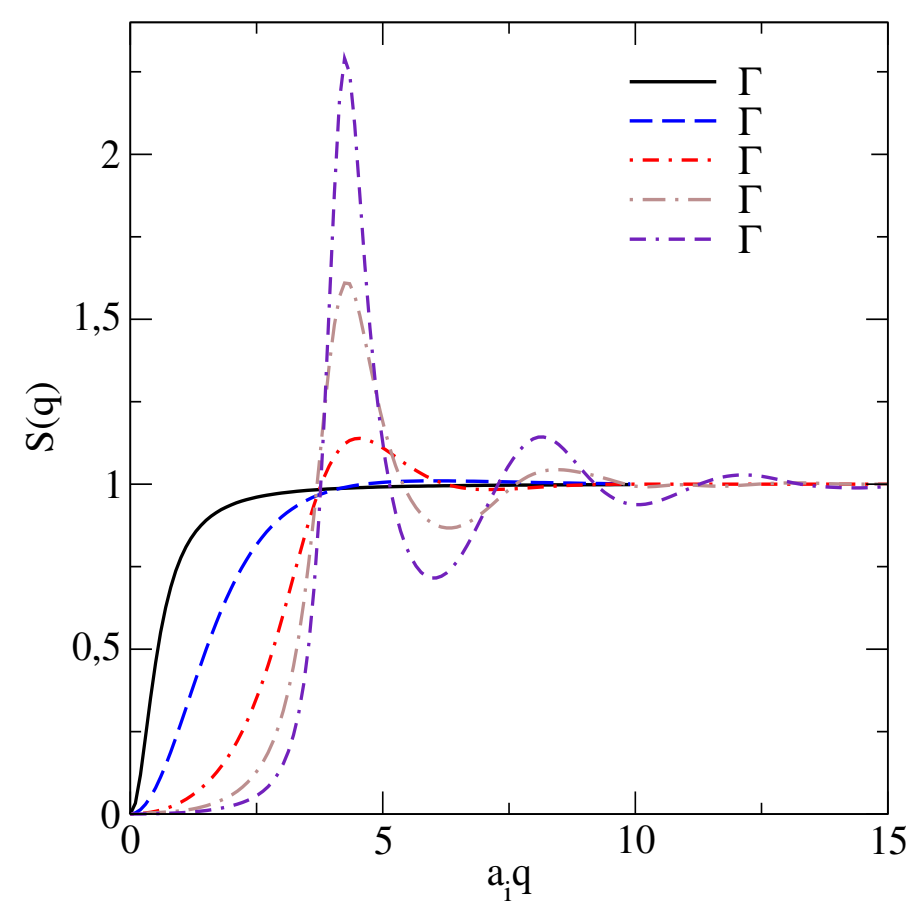

Figure 3: Dependence of the structure function of one-component plasma on the magnitude of momentum transfer $q$ in units of inverse $a_{i}$. For $\Gamma \geq 2$ the structure function is taken from Monte-Carlo calculations of Galam and Hansen [16]. For $\Gamma<2$ we obtain the structure function from the analytical expressions provided by Tamashiro et al. [17].

the collision intergral. This is the case, for example, when ions form a solid lattice and, therefore, Umklapp $e-e$ processes are allowed, or in the case of thermal conduction and shear stresses when the $e-e$ collisions contribute to the dissipation. Finally, we note that in order to account for the finite size of the nuclei we have multiplied the transition probability in Eq. (3.13) by the standard expression for the nuclear formfactor [15]

$$
F(q)=-3 \frac{q r_{c} \cos \left(q r_{c}\right)-\sin \left(q r_{c}\right)}{\left(q r_{c}\right)^{3}}
$$

where $r_{c}$ is the charge radius of the nucleus given by $r_{c}=1.15 A^{1 / 3} \mathrm{fm}$.

\section{Results}

For the numerical computations we need to specify the ion structure function $S(q)$. We assume that only one sort of ions exists at a given density, so that the structure functions of one-component plasma (OCP) can be used. These has been extensively computed using various numerical methods. We adopt the Monte-Carlo results of Galam and Hansen [16] for Coulomb OCP provided in tabular form and set a two-dimension spline function in the space spanned by the magnitude of the momentum transfer $q$ and the plasma parameter $\Gamma$. In the low- $\Gamma$ regime $(\Gamma \leq 2)$ we used the analytical (leading order) expressions derived by Tamashiro et al. [17] for Coulomb OCP derived 
using density functional methods. The resulting structure functions for various values of the plasma parameter $\Gamma$ are shown in Fig. 3 as a function of the dimensionless parameter $a_{i} q$, where $a_{i}$ is the ion-radius as defined after Eq. (2.1). It is seen that the structure factor universally suppresses the contribution from small- $q$ scattering. The suppression sets in for larger $q$ at larger values of $\Gamma$. The large- $q$ asymptotics is independent of $\Gamma$ as $S(q) \rightarrow 1$. The major difference arises for intermediate values of $q$ where the structure factor oscillates and the amplitude of oscillations increases with the value of $\Gamma$ parameter. The screening of longitudinal and transverse interactions is determined by the corresponding components of the polarization tensor. While expression (3.13) is exact with respect to the form of the polarization tensor, in the numerical calculations we use the hard-thermal-loop approximation and next-to-leading expansion in $x=\omega / q$. For the real and imaginary parts of the polarization tensor we find

$$
\Pi_{l}(q, \omega)=q_{D}^{2} \chi_{l}, \quad \Pi_{t}(q, \omega)=q_{D}^{2} \chi_{t},
$$

where $q_{D}$ is the Debye wave-length and the susceptibilities to order $O\left(x^{2}\right)$ are given by

$$
\begin{aligned}
& \operatorname{Re} \chi_{l}(q, \omega)=1-\frac{x^{2}}{\bar{v}^{2}}, \quad \operatorname{Im} \chi_{l}(q, \omega)=-\frac{\pi x}{2 \bar{v}}, \\
& \operatorname{Re} \chi_{t}(q, \omega)=x^{2}, \quad \operatorname{Im} \chi_{t}(q, \omega)=\frac{\pi}{4} x \bar{v}
\end{aligned}
$$

where $\bar{v}$ is the electrons average velocity. Because the terms containing $\bar{v}$ are small as well as electrons are ultra-relativistic in the most of the regime of interest we approximate $\bar{v}=1$ in our numerical calculations. For the longitudinal piece of the polarization tensor the screening is finite in the static case $x=0$, while it vanishes for the transverse piece as $\Pi_{t}(q, \omega) \propto x^{2}$, hence the purely dynamical nature of the transverse screening.

In the zero-temperature limit Eq. (3.12) simplifies via the substitution $T \partial f^{0} / \partial \varepsilon=-f^{0}(1-$ $\left.f^{0}\right) \rightarrow-T \delta\left(\varepsilon-\varepsilon_{F}\right)$, i.e.,

$$
\sigma=\frac{e^{2}}{3 \pi^{2}} \int_{m}^{\infty} d \varepsilon \frac{p^{3}}{\varepsilon} \tau(\varepsilon) \delta\left(\varepsilon-\varepsilon_{F}\right)=\frac{n_{e} e^{2} \tau_{F}}{\varepsilon_{F}}
$$

where $\tau_{F}$ is the relaxation time (3.13) taken on the Fermi surface in the $T=0$ limit

$$
\tau_{F}^{-1} \equiv \tau^{-1}\left(\varepsilon_{F}\right)=\frac{4}{3 \pi} Z e^{4} \varepsilon_{F} \int_{0}^{2 p_{F}} d q \frac{q^{3}}{\left|q^{2}+\Pi_{l}\right|^{2}}\left(1-\frac{q^{2}}{4 \varepsilon_{F}^{2}}\right) S(q) F^{2}(q),
$$

where employed the charge neutrality condition $n_{e}=Z n_{i}$. Neglecting the screening $\left(\Pi_{l} \rightarrow 0\right)$ and the nuclear formfactor $[F(q) \rightarrow 1]$ we obtain from (4.5)

$$
\tau_{F}^{-1}=\frac{4 Z e^{4} \varepsilon_{F}}{3 \pi} \int_{0}^{2 p_{F}} \frac{d q}{q}\left(1-\frac{q^{2}}{4 \varepsilon_{F}^{2}}\right) S(q),
$$

which coincides with Eqs. (9) and (11) of Ref. [6].

With the input described above we have evaluated the relaxation time for electron scattering off the ions using Eq. (3.13) and then the components of the conductivity tensor according to Eq. (3.10). Here we demonstrate selected results, while our complete results are discussed elsewhere [12]. The conductivity as a function of temperature is shown in Fig. 4 for carbon ${ }^{12} \mathrm{C}$ and 


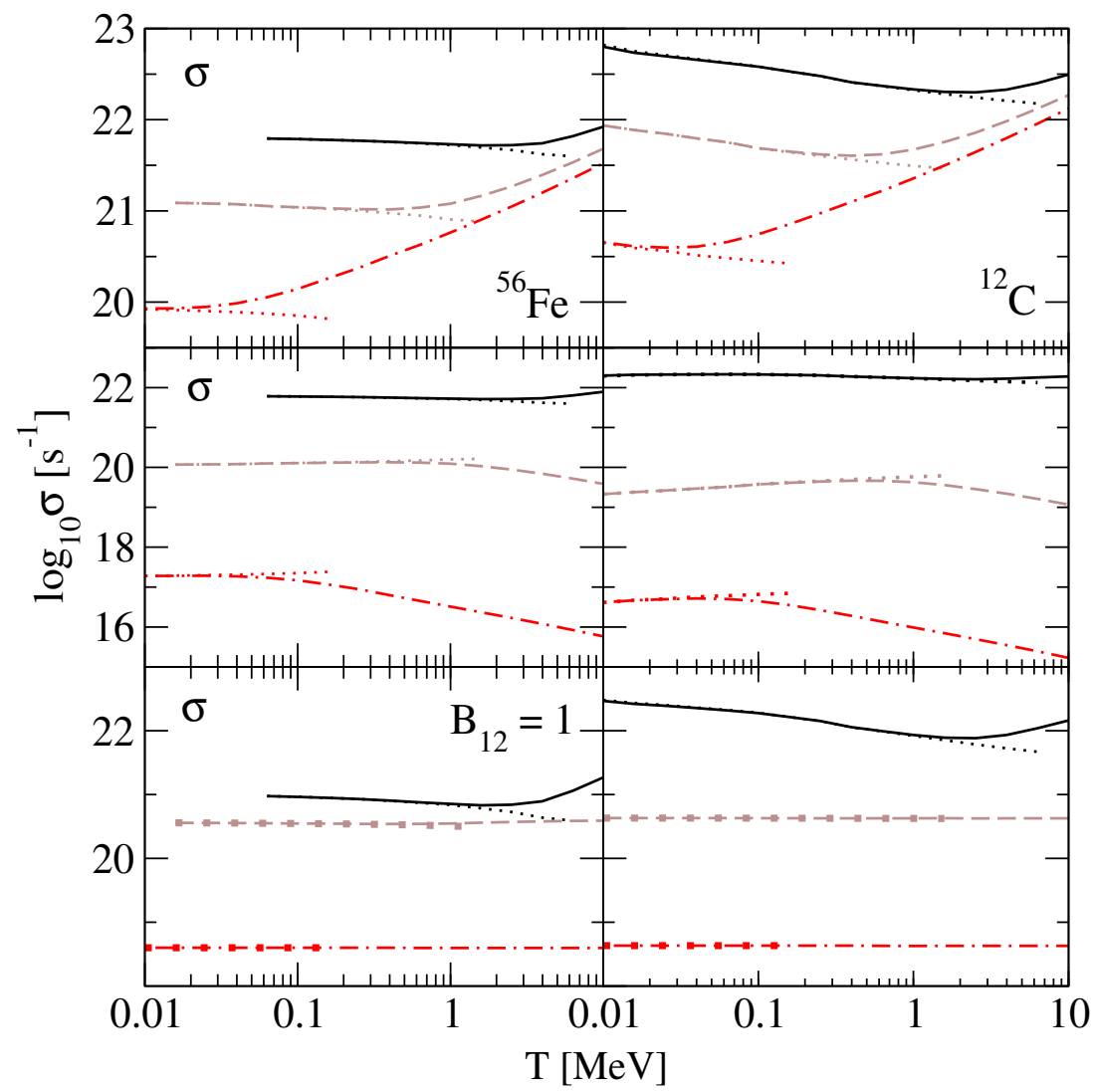

Figure 4: Dependence of the electrical scalar conductivity $\sigma$ (upper panel) and tensor components $\sigma_{0}$ (middle panel) and $\sigma_{1}$ (lower panel) on temperature for three densities $\log _{10} \rho=10$ (solid $\left.\operatorname{lines}\right), \log _{10} \rho=8$ (dashed lines) and $\log _{10} \rho=6$ (dash-dotted lines). The left column contains results for ${ }^{56} \mathrm{Fe}$, the right one for ${ }^{12} \mathrm{C}$. The dotted lines (symbols in the two lower panels) associated with each line show the same, but are evaluate from the zero-temperature Drude formula. The magnetic field is fixed at $B_{12}=1$.

iron ${ }^{56} \mathrm{Fe}$ nuclei. The magnitude of the magnetic field is fixed to $B_{12}=1$, where $B_{12}$ is the magnetic field in units of $10^{12} \mathrm{G}$. The full results are compared to the case where the conductivity is evaluated from the Drude formula (4.4), which is shown by dotted lines. The deviation from the zero temperature result are visible for temperatures in the range $0.1-1 \mathrm{MeV}\left(\mathrm{MeV}=1.16 \times 10^{10} \mathrm{~K}\right)$ when the density is varied from $10^{6}$ to $10^{10} \mathrm{~g} \mathrm{~cm}^{-3}$. It is seen that the $\sigma$ component of conductivity has a minimum as a function of temperature: the low-temperature decrease is replace by a power-law increase with increasing temperature. This increase can be understood in terms of the smearing of the Fermi surface by temperature which makes more electrons available for conduction. The minimum of the conductivity is one of the key findings of our work.

The same as in Fig. 4 but as a function of density for fixed temperature values is shown in Fig. 5. The scalar conductivity and $\sigma_{0}$ component are increasing functions of density and depend strongly on the temperature in the low-density limit, which is associated with lifting of the degeneracy as the temperature is increased. The behaviour of $\sigma_{1}$ is reversed: it is almost independent of temperature and has a maximum. This is the consequence of the different scaling of the compo- 


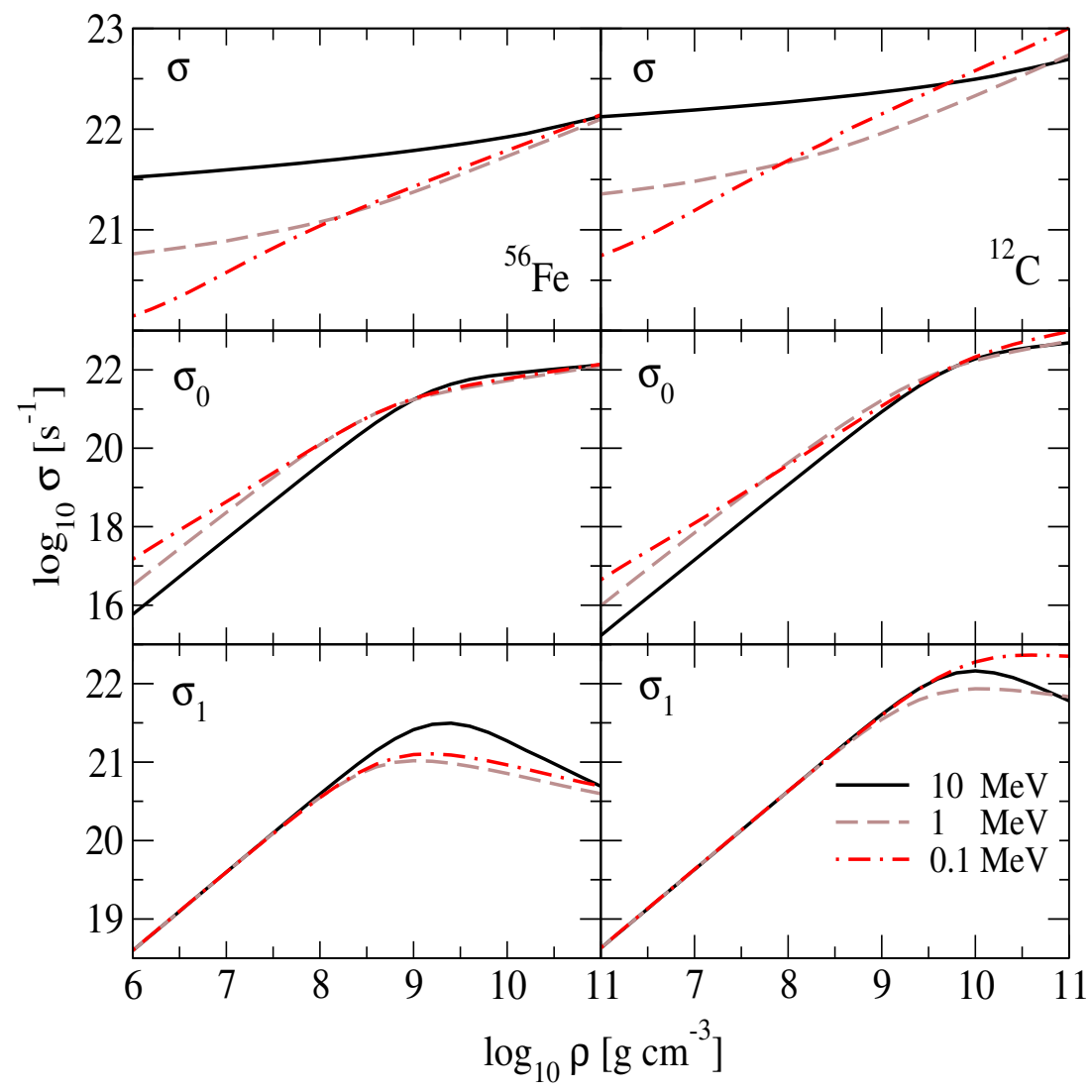

Figure 5: Dependence of the electrical scalar conductivity $\sigma$ (upper panel) and tensor components $\sigma_{0}$ (middle panel) and $\sigma_{1}$ (lower panel) on density for three temperatures $T=10 \mathrm{MeV}$ (solid lines), $T=1 \mathrm{MeV}$ (dashed lines) and $T=0.1 \mathrm{MeV}$ (dash-dotted lines) in the case of ${ }^{56} \mathrm{Fe}$ (left) and $C^{12} \mathrm{C}$ (right) nuclei and magnetic field $B_{12}=1$.

nents of the conductivity tensor with $\omega_{c} \tau$ parameter, which describes the effects of magnetic field. Our results in the cases of matter composed of ${ }^{12} \mathrm{C}$ or matter composed of series of nuclei (when the composition varies with density) show the same general trends as for ${ }^{56} \mathrm{Fe}$. The differences between these cases are quantitative and are discussed in detailed in Ref. [12].

\section{Conclusions}

In this contribution we gave an overview of our current work on the conductivity of dense matter in the envelopes of neutron stars at non-zero temperature. One ingredient of our effort is the formulation of the transport in a manner which allows us to include the dynamical screening exactly, provided that the polarization tensor of electrons (or equivalently the self-energies of QED photons) in plasma can be computed to desired accuracy. Here we employed the results based on the hard-thermal-loop approximation and low-frequency expansion appropriate at not very high temperatures. We have shown that for electron-ion scattering the dynamical screening is suppressed parametrically by a factor $M / T$, but we anticipate that its effect would be substantial in the cases (a) of high temperatures and presence of light clusters, (b) of low temperatures, where the Umklapp 
processes with $e$-e scattering are important, (c) of transport processes where $e$-e scattering may be dominant from the outset, such as the thermal conductivity and shear viscosity.

Our numerical results show that the scalar conductivity (no anisotropy due to the $B$-field) has a minimum as a function of temperature, with a power-law decrease at low-temperatures and a powerlaw increase at higher temperatures. The range of validity of the zero-temperature Drude formula extends from low temperatures up to $0.1-1 \mathrm{MeV}\left(10^{9}-10^{10} \mathrm{~K}\right)$, where the lower of these bounds corresponds to density $\rho \sim 10^{6} \mathrm{~g} \mathrm{~cm}^{-3}$ and the upper one to $\rho \sim 10^{10} \mathrm{~g} \mathrm{~cm}^{-3}$. The behaviour of the off-diagonal $\sigma_{1}$ component of the conductivity tensor is similar to the one described above except at low densities, where it remains almost constant. Finally the $\sigma_{0}$ component shows strongly density dependent behaviour: for large densities (high degeneracy) it behaves analogous to $\sigma$, but shows the inverse trend for low-densities which is associated with the transition from the regime $\omega_{c} \tau<1$ to $\omega_{c} \tau>1$.

\section{Acknowledgments}

We thank M. Alford, H. Nishimura, L. Rezzolla and D. Rischke for discussions. This work was supported by the HGS-HIRe graduate program at Frankfurt University (A. H.), by the Deutsche Forschungsgemeinschaft's Grant No. SE 1836/3-1 (A. S.) and by the NewCompStar COST Action MP1304. We thank the Volkswagen Stiftung for the support of the 2015 edition of the conference series "The Modern Physics of Compact Stars and Relativistic Gravity”.

\section{References}

[1] L. Mestel and F. Hoyle, On the thermal conductivity in dense stars, Proceedings of the Cambridge Philosophical Society 46 (1950) 331.

[2] T. D. Lee, Hydrogen Content and Energy-Productive Mechanism of White Dwarfs., ApJ 111 (1950) 625.

[3] A. A. Abrikosov, The conductivity of strongly compressed matter, Soviet Physics JETP 18 (1964) 1399.

[4] E. Flowers and N. Itoh, Transport properties of dense matter, ApJ 206 (1976) 218.

[5] D. G. Yakovlev and V. A. Urpin, Thermal and Electrical Conductivity in White Dwarfs and Neutron Stars, Soviet Ast. 24 (1980) 303.

[6] R. Nandkumar and C. J. Pethick, Transport coefficients of dense matter in the liquid metal regime, MNRAS 209 (1984) 511.

[7] N. Itoh, S. Mitake, H. Iyetomi and S. Ichimaru, Electrical and thermal conductivities of dense matter in the liquid metal phase. I - High-temperature results, ApJ 273 (1983) 774.

[8] A. Y. Potekhin, D. A. Baiko, P. Haensel and D. G. Yakovlev, Transport properties of degenerate electrons in neutron star envelopes and white dwarf cores, A\&A 346 (1999) 345, [astro-ph/9903127].

[9] N. Itoh, S. Uchida, Y. Sakamoto, Y. Kohyama and S. Nozawa, The Second Born Corrections to the Electrical and Thermal Conductivities of Dense Matter in the Liquid Metal Phase, ApJ 677 (2008) 495, [0 708 .2967]. 
[10] I. Easson and C. J. Pethick, Magnetohydrodynamics of neutron star interiors, ApJ 227 (1979) 995.

[11] D. M. Sedrakyan and A. K. Avetisyan, Magnetohydrodynamics of Plasma in the Crust of a Neutron Star, Astrophysics 26 (1987) 295.

[12] A. Harutyunyan and A. Sedrakian, Electrical conductivity of warm neutron star crust in magnetic fields, Arxiv eprint: [1605.07612].

[13] H. Heiselberg and C. J. Pethick, Transport and relaxation in degenerate quark plasmas, Phys. Rev. D 48 (1993) 2916.

[14] M. G. Alford, H. Nishimura and A. Sedrakian, Transport coefficients of two-flavor superconducting quark matter, Phys. Rev. C 90 (2014) 055205, [1 408 . 4999].

[15] N. Itoh, Y. Kohyama, N. Matsumoto and M. Seki, Electrical and thermal conductivities of dense matter in the crystalline lattice phase, ApJ 285 (1984) 758.

[16] S. Galam and J.-P. Hansen, Statistical mechanics of dense ionized matter. VI. Electron screening corrections to the thermodynamic properties of the one-component plasma, Phys. Rev. A 14 (1976) 816.

[17] M. N. Tamashiro, Y. Levin and M. C. Barbosa, The one-component plasma: a conceptual approach, Physica A Statistical Mechanics and its Applications 268 (1999) 24, [cond-mat/9810213].

\section{Supplemental material}

Below we present numerical tables for the conductivities $\log _{10} \sigma, \log _{10} \sigma_{0}$ and $\log _{10} \sigma_{1}$ (in units of $\mathrm{s}^{-1}$ ) for various values of magnetic field (in units of $10^{12} \mathrm{G}$ ) for sets of values of density [g $\left.\mathrm{cm}^{-3}\right]$ and temperature $[\mathrm{MeV}]$. The tables are provided for three types of composition of matter: ${ }^{12} \mathrm{C}$ nuclei, ${ }^{56} \mathrm{Fe}$ nuclei, and density-dependent composition as indicated in Fig. 2. Analytical fits to these results with relative error $\leq 10 \%$ can be found in Ref. [12]. 
Table 1: $\log \sigma$ for ${ }^{12} \mathrm{C}$

\begin{tabular}{|c|c|c|c|c|c|c|c|c|c|c|c|}
\hline $\log \rho$ & $\log T=-1.0$ & $\log T=-0.8$ & $\log T=-0.6$ & $\log T=-0.4$ & $\log T=-0.2$ & $\log T=0.0$ & $\log T=0.2$ & $\log T=0.4$ & $\log T=0.6$ & $\log T=0.8$ & $\log T=1.0$ \\
\hline 6.0 & 20.745 & 20.853 & 20.973 & 21.100 & 21.222 & 21.356 & 21.498 & 21.646 & 21.798 & 21.957 & 22.123 \\
\hline 6.2 & 20.816 & 20.905 & 21.013 & 21.131 & 21.251 & 21.378 & 21.518 & 21.663 & 21.814 & 21.970 & 22.136 \\
\hline 6.4 & 20.898 & 20.965 & 21.057 & 21.165 & 21.281 & 21.401 & 21.538 & 21.681 & 21.830 & 21.985 & 22.149 \\
\hline 6.6 & 20.990 & 21.033 & 21.106 & 21.202 & 21.313 & 21.426 & 21.559 & 21.699 & 21.846 & 22.000 & 22.162 \\
\hline 6.8 & 21.089 & 21.110 & 21.163 & 21.244 & 21.346 & 21.452 & 21.581 & 21.719 & 21.864 & 22.015 & 22.176 \\
\hline 7.0 & 21.192 & 21.193 & 21.226 & 21.290 & 21.381 & 21.482 & 21.605 & 21.739 & 21.882 & 22.031 & 22.190 \\
\hline 7.2 & 21.296 & 21.283 & 21.296 & 21.342 & 21.420 & 21.518 & 21.630 & 21.760 & 21.900 & 22.048 & 22.205 \\
\hline 7.4 & 21.398 & 21.375 & 21.372 & 21.400 & 21.462 & 21.553 & 21.656 & 21.783 & 21.920 & 22.065 & 22.220 \\
\hline 7.6 & 21.498 & 21.467 & 21.452 & 21.463 & 21.510 & 21.589 & 21.684 & 21.806 & 21.940 & 22.083 & 22.236 \\
\hline 7.8 & 21.595 & 21.560 & 21.535 & 21.532 & 21.562 & 21.629 & 21.719 & 21.831 & 21.961 & 22.102 & 22.253 \\
\hline 8.0 & 21.688 & 21.650 & 21.620 & 21.606 & 21.620 & 21.672 & 21.756 & 21.858 & 21.984 & 22.121 & 22.270 \\
\hline 8.2 & 21.779 & 21.740 & 21.705 & 21.682 & 21.683 & 21.720 & 21.794 & 21.886 & 22.008 & 22.142 & 22.288 \\
\hline 8.4 & 21.867 & 21.827 & 21.789 & 21.761 & 21.751 & 21.773 & 21.833 & 21.920 & 22.033 & 22.163 & 22.306 \\
\hline 8.6 & 21.979 & 21.913 & 21.874 & 21.841 & 21.822 & 21.831 & 21.877 & 21.958 & 22.059 & 22.186 & 22.326 \\
\hline 8.8 & 22.065 & 21.998 & 21.958 & 21.921 & 21.896 & 21.893 & 21.925 & 21.996 & 22.088 & 22.209 & 22.346 \\
\hline 9.0 & 22.151 & 22.082 & 22.041 & 22.002 & 21.972 & 21.959 & 21.978 & 22.036 & 22.121 & 22.234 & 22.368 \\
\hline 9.2 & 22.237 & 22.191 & 22.124 & 22.084 & 22.050 & 22.029 & 22.035 & 22.080 & 22.159 & 22.261 & 22.390 \\
\hline 9.4 & 22.323 & 22.274 & 22.207 & 22.166 & 22.129 & 22.102 & 22.097 & 22.128 & 22.198 & 22.290 & 22.414 \\
\hline 9.6 & 22.410 & 22.358 & 22.289 & 22.247 & 22.208 & 22.177 & 22.163 & 22.181 & 22.238 & 22.323 & 22.439 \\
\hline 9.8 & 22.496 & 22.443 & 22.396 & 22.329 & 22.289 & 22.254 & 22.233 & 22.238 & 22.282 & 22.362 & 22.466 \\
\hline 10.0 & 22.581 & 22.529 & 22.479 & 22.411 & 22.369 & 22.332 & 22.305 & 22.300 & 22.330 & 22.401 & 22.495 \\
\hline 10.2 & 22.665 & 22.615 & 22.563 & 22.492 & 22.451 & 22.411 & 22.380 & 22.366 & 22.383 & 22.441 & 22.528 \\
\hline 10.4 & 22.747 & 22.701 & 22.648 & 22.600 & 22.532 & 22.492 & 22.457 & 22.435 & 22.441 & 22.486 & 22.568 \\
\hline 10.6 & 22.830 & 22.788 & 22.734 & 22.683 & 22.614 & 22.573 & 22.535 & 22.508 & 22.503 & 22.534 & 22.608 \\
\hline 10.8 & 22.915 & 22.873 & 22.821 & 22.768 & 22.697 & 22.654 & 22.614 & 22.583 & 22.569 & 22.588 & 22.649 \\
\hline 11.0 & 23.002 & 22.959 & 22.910 & 22.854 & 22.805 & 22.737 & 22.695 & 22.660 & 22.639 & 22.646 & 22.694 \\
\hline
\end{tabular}

Table 2: $\log \sigma_{0}$ for ${ }^{12} \mathrm{C}$ at $B_{12}=1$

\begin{tabular}{|c|c|c|c|c|c|c|c|c|c|c|c|}
\hline $\log \rho$ & $\log T=-1.0$ & $\log T=-0.8$ & $\log T=-0.6$ & $\log T=-0.4$ & $\log T=-0.2$ & $\log T=0.0$ & $\log T=0.2$ & $\log T=0.4$ & $\log T=0.6$ & $\log T=0.8$ & $\log T=1.0$ \\
\hline 6.0 & 16.648 & 16.551 & 16.423 & 16.279 & 16.132 & 15.987 & 15.843 & 15.696 & 15.546 & 15.390 & 15.227 \\
\hline 6.2 & 16.947 & 16.878 & 16.771 & 16.641 & 16.503 & 16.363 & 16.222 & 16.078 & 15.929 & 15.776 & 15.613 \\
\hline 6.4 & 17.238 & 17.195 & 17.112 & 16.999 & 16.871 & 16.736 & 16.599 & 16.458 & 16.312 & 16.160 & 15.999 \\
\hline 6.6 & 17.522 & 17.504 & 17.445 & 17.352 & 17.236 & 17.108 & 16.976 & 16.838 & 16.694 & 16.544 & 16.385 \\
\hline 6.8 & 17.805 & 17.806 & 17.771 & 17.699 & 17.597 & 17.478 & 17.351 & 17.216 & 17.075 & 16.928 & 16.770 \\
\hline 7.0 & 18.090 & 18.104 & 18.091 & 18.040 & 17.955 & 17.845 & 17.724 & 17.594 & 17.455 & 17.310 & 17.155 \\
\hline 7.2 & 18.378 & 18.402 & 18.405 & 18.375 & 18.307 & 18.211 & 18.096 & 17.970 & 17.835 & 17.692 & 17.539 \\
\hline 7.4 & 18.672 & 18.702 & 18.716 & 18.704 & 18.655 & 18.572 & 18.465 & 18.345 & 18.213 & 18.073 & 17.923 \\
\hline 7.6 & 18.969 & 19.003 & 19.027 & 19.028 & 18.997 & 18.929 & 18.832 & 18.718 & 18.590 & 18.453 & 18.306 \\
\hline 7.8 & 19.271 & 19.307 & 19.336 & 19.349 & 19.333 & 19.281 & 19.196 & 19.089 & 18.966 & 18.832 & 18.688 \\
\hline 8.0 & 19.575 & 19.613 & 19.646 & 19.667 & 19.664 & 19.628 & 19.556 & 19.457 & 19.340 & 19.210 & 19.069 \\
\hline 8.2 & 19.882 & 19.921 & 19.956 & 19.982 & 19.990 & 19.968 & 19.910 & 19.821 & 19.711 & 19.586 & 19.448 \\
\hline 8.4 & 20.190 & 20.229 & 20.265 & 20.295 & 20.310 & 20.300 & 20.257 & 20.179 & 20.078 & 19.959 & 19.826 \\
\hline 8.6 & 20.473 & 20.535 & 20.571 & 20.602 & 20.622 & 20.622 & 20.592 & 20.530 & 20.438 & 20.328 & 20.201 \\
\hline 8.8 & 20.778 & 20.837 & 20.872 & 20.903 & 20.925 & 20.931 & 20.913 & 20.865 & 20.787 & 20.688 & 20.570 \\
\hline 9.0 & 21.077 & 21.133 & 21.164 & 21.191 & 21.213 & 21.222 & 21.213 & 21.180 & 21.119 & 21.035 & 20.929 \\
\hline 9.2 & 21.367 & 21.399 & 21.441 & 21.464 & 21.481 & 21.489 & 21.486 & 21.466 & 21.425 & 21.360 & 21.272 \\
\hline 9.4 & 21.642 & 21.669 & 21.699 & 21.712 & 21.722 & 21.725 & 21.723 & 21.713 & 21.692 & 21.652 & 21.589 \\
\hline 9.6 & 21.897 & 21.915 & 21.929 & 21.932 & 21.931 & 21.927 & 21.922 & 21.918 & 21.915 & 21.901 & 21.869 \\
\hline 9.8 & 22.128 & 22.132 & 22.131 & 22.120 & 22.108 & 22.095 & 22.084 & 22.081 & 22.090 & 22.101 & 22.101 \\
\hline 10.0 & 22.329 & 22.320 & 22.305 & 22.278 & 22.257 & 22.235 & 22.217 & 22.210 & 22.223 & 22.252 & 22.280 \\
\hline 10.2 & 22.500 & 22.480 & 22.453 & 22.410 & 22.382 & 22.353 & 22.328 & 22.316 & 22.325 & 22.362 & 22.410 \\
\hline 10.4 & 22.644 & 22.616 & 22.580 & 22.545 & 22.491 & 22.457 & 22.427 & 22.408 & 22.410 & 22.445 & 22.506 \\
\hline 10.6 & 22.767 & 22.735 & 22.693 & 22.650 & 22.590 & 22.552 & 22.518 & 22.492 & 22.487 & 22.514 & 22.577 \\
\hline 10.8 & 22.876 & 22.842 & 22.796 & 22.748 & 22.682 & 22.642 & 22.605 & 22.574 & 22.561 & 22.577 & 22.634 \\
\hline 11.0 & 22.979 & 22.940 & 22.894 & 22.842 & 22.796 & 22.730 & 22.690 & 22.655 & 22.635 & 22.641 & 22.687 \\
\hline
\end{tabular}

Table 3: $\log \sigma_{0}$ for ${ }^{12} \mathrm{C}$ at $B_{12}=10$

\begin{tabular}{|c|c|c|c|c|c|c|c|c|c|c|c|}
\hline $\log \rho$ & $\log T=-1.0$ & $\log T=-0.8$ & $\log T=-0.6$ & $\log T=-0.4$ & $\log T=-0.2$ & $\log T=0.0$ & $\log T=0.2$ & $\log T=0.4$ & $\log T=0.6$ & $\log T=0.8$ & $\log T=1.0$ \\
\hline 6.0 & 14.648 & 14.551 & 14.423 & 14.280 & 14.133 & 13.988 & 13.842 & 13.696 & 13.546 & 13.390 & 13.227 \\
\hline 6.2 & 14.948 & 14.878 & 14.771 & 14.642 & 14.503 & 14.363 & 14.221 & 14.078 & 13.929 & 13.776 & 13.613 \\
\hline 6.4 & 15.238 & 15.195 & 15.112 & 14.999 & 14.871 & 14.736 & 14.599 & 14.458 & 14.312 & 14.160 & 13.999 \\
\hline 6.6 & 15.523 & 15.504 & 15.446 & 15.352 & 15.236 & 15.108 & 14.975 & 14.838 & 14.694 & 14.544 & 14.385 \\
\hline 6.8 & 15.806 & 15.806 & 15.772 & 15.700 & 15.598 & 15.478 & 15.351 & 15.216 & 15.075 & 14.928 & 14.770 \\
\hline 7.0 & 16.090 & 16.105 & 16.092 & 16.041 & 15.955 & 15.846 & 15.724 & 15.594 & 15.455 & 15.310 & 15.155 \\
\hline 7.2 & 16.379 & 16.403 & 16.406 & 16.376 & 16.308 & 16.212 & 16.096 & 15.970 & 15.835 & 15.692 & 15.539 \\
\hline 7.4 & 16.672 & 16.703 & 16.718 & 16.705 & 16.656 & 16.573 & 16.466 & 16.345 & 16.213 & 16.073 & 15.923 \\
\hline 7.6 & 16.970 & 17.005 & 17.028 & 17.031 & 16.999 & 16.931 & 16.833 & 16.719 & 16.591 & 16.454 & 16.306 \\
\hline 7.8 & 17.273 & 17.310 & 17.339 & 17.353 & 17.337 & 17.285 & 17.199 & 17.090 & 16.967 & 16.833 & 16.688 \\
\hline 8.0 & 17.579 & 17.617 & 17.651 & 17.673 & 17.671 & 17.634 & 17.561 & 17.460 & 17.342 & 17.212 & 17.069 \\
\hline 8.2 & 17.888 & 17.928 & 17.964 & 17.992 & 18.000 & 17.978 & 17.920 & 17.828 & 17.716 & 17.589 & 17.450 \\
\hline 8.4 & 18.199 & 18.240 & 18.278 & 18.310 & 18.327 & 18.318 & 18.274 & 18.193 & 18.088 & 17.965 & 17.830 \\
\hline 8.6 & 18.487 & 18.553 & 18.593 & 18.628 & 18.652 & 18.654 & 18.623 & 18.556 & 18.457 & 18.340 & 18.208 \\
\hline 8.8 & 18.801 & 18.868 & 18.909 & 18.946 & 18.975 & 18.986 & 18.969 & 18.914 & 18.824 & 18.713 & 18.586 \\
\hline 9.0 & 19.115 & 19.184 & 19.225 & 19.264 & 19.297 & 19.315 & 19.309 & 19.268 & 19.189 & 19.085 & 18.962 \\
\hline 9.2 & 19.429 & 19.475 & 19.542 & 19.582 & 19.617 & 19.642 & 19.645 & 19.617 & 19.551 & 19.454 & 19.337 \\
\hline 9.4 & 19.742 & 19.791 & 19.858 & 19.899 & 19.936 & 19.965 & 19.977 & 19.961 & 19.908 & 19.820 & 19.709 \\
\hline 9.6 & 20.055 & 20.106 & 20.175 & 20.215 & 20.254 & 20.287 & 20.305 & 20.300 & 20.260 & 20.182 & 20.079 \\
\hline 9.8 & 20.368 & 20.419 & 20.465 & 20.531 & 20.570 & 20.605 & 20.628 & 20.632 & 20.604 & 20.540 & 20.444 \\
\hline 10.0 & 20.680 & 20.731 & 20.779 & 20.844 & 20.883 & 20.918 & 20.945 & 20.956 & 20.940 & 20.889 & 20.804 \\
\hline 10.2 & 20.993 & 21.040 & 21.089 & 21.154 & 21.191 & 21.226 & 21.255 & 21.270 & 21.263 & 21.226 & 21.153 \\
\hline 10.4 & 21.304 & 21.346 & 21.394 & 21.436 & 21.493 & 21.526 & 21.553 & 21.570 & 21.570 & 21.545 & 21.488 \\
\hline 10.6 & 21.610 & 21.647 & 21.691 & 21.732 & 21.783 & 21.811 & 21.835 & 21.851 & 21.855 & 21.839 & 21.799 \\
\hline 10.8 & 21.907 & 21.940 & 21.978 & 22.015 & 22.057 & 22.078 & 22.095 & 22.107 & 22.110 & 22.101 & 22.077 \\
\hline 11.0 & 22.192 & 22.221 & 22.250 & 22.278 & 22.299 & 22.320 & 22.328 & 22.331 & 22.330 & 22.325 & 22.315 \\
\hline
\end{tabular}


Table 4: $\log \sigma_{0}$ for ${ }^{12} \mathrm{C}$ at $B_{12}=100$

\begin{tabular}{|c|c|c|c|c|c|c|c|c|c|c|c|}
\hline $\log \rho$ & $\log T=-1.0$ & $\log T=-0.8$ & $\log T=-0.6$ & $\log T=-0.4$ & $\log T=-0.2$ & $\log T=0.0$ & $\log T=0.2$ & $\log T=0.4$ & $\log T=0.6$ & $\log T=0.8$ & $\log T=1.0$ \\
\hline 6.0 & 12.648 & 12.551 & 12.423 & 12.280 & 12.133 & 11.988 & 11.843 & 11.696 & 11.546 & 11.390 & 11.227 \\
\hline 6.2 & 12.948 & 12.878 & 12.771 & 12.642 & 12.503 & 12.363 & 12.222 & 12.078 & 11.929 & 11.776 & 11.613 \\
\hline 6.4 & 13.238 & 13.195 & 13.112 & 12.999 & 12.871 & 12.736 & 12.599 & 12.458 & 12.312 & 12.160 & 11.999 \\
\hline 6.6 & 13.523 & 13.504 & 13.446 & 13.352 & 13.236 & 13.108 & 12.976 & 12.838 & 12.694 & 12.544 & 12.385 \\
\hline 6.8 & 13.806 & 13.806 & 13.772 & 13.700 & 13.598 & 13.478 & 13.351 & 13.216 & 13.075 & 12.928 & 12.770 \\
\hline 7.0 & 14.090 & 14.105 & 14.092 & 14.041 & 13.955 & 13.846 & 13.724 & 13.594 & 13.455 & 13.310 & 13.155 \\
\hline 7.2 & 14.379 & 14.403 & 14.406 & 14.376 & 14.308 & 14.212 & 14.096 & 13.970 & 13.835 & 13.692 & 13.539 \\
\hline 7.4 & 14.672 & 14.703 & 14.718 & 14.705 & 14.656 & 14.573 & 14.466 & 14.345 & 14.213 & 14.073 & 13.923 \\
\hline 7.6 & 14.970 & 15.005 & 15.028 & 15.031 & 14.999 & 14.931 & 14.833 & 14.719 & 14.591 & 14.454 & 14.306 \\
\hline 7.8 & 15.273 & 15.310 & 15.339 & 15.353 & 15.337 & 15.285 & 15.199 & 15.090 & 14.967 & 14.833 & 14.688 \\
\hline 8.0 & 15.579 & 15.618 & 15.651 & 15.673 & 15.671 & 15.634 & 15.561 & 15.460 & 15.342 & 15.212 & 15.069 \\
\hline 8.2 & 15.888 & 15.928 & 15.964 & 15.992 & 16.000 & 15.979 & 15.920 & 15.828 & 15.716 & 15.589 & 15.450 \\
\hline 8.4 & 16.200 & 16.240 & 16.278 & 16.310 & 16.327 & 16.319 & 16.274 & 16.193 & 16.088 & 15.965 & 15.830 \\
\hline 8.6 & 16.487 & 16.553 & 16.593 & 16.628 & 16.652 & 16.655 & 16.624 & 16.556 & 16.457 & 16.340 & 16.208 \\
\hline 8.8 & 16.801 & 16.869 & 16.909 & 16.947 & 16.976 & 16.987 & 16.969 & 16.914 & 16.825 & 16.714 & 16.586 \\
\hline 9.0 & 17.115 & 17.185 & 17.226 & 17.265 & 17.298 & 17.316 & 17.310 & 17.269 & 17.190 & 17.085 & 16.962 \\
\hline 9.2 & 17.429 & 17.476 & 17.543 & 17.583 & 17.619 & 17.644 & 17.647 & 17.619 & 17.553 & 17.455 & 17.337 \\
\hline 9.4 & 17.743 & 17.792 & 17.860 & 17.901 & 17.939 & 17.969 & 17.981 & 17.965 & 17.911 & 17.823 & 17.711 \\
\hline 9.6 & 18.057 & 18.108 & 18.178 & 18.219 & 18.259 & 18.292 & 18.312 & 18.306 & 18.266 & 18.188 & 18.082 \\
\hline 9.8 & 18.371 & 18.423 & 18.470 & 18.538 & 18.578 & 18.614 & 18.640 & 18.644 & 18.616 & 18.550 & 18.452 \\
\hline 10.0 & 18.686 & 18.738 & 18.788 & 18.856 & 18.897 & 18.935 & 18.965 & 18.978 & 18.962 & 18.909 & 18.819 \\
\hline 10.2 & 19.002 & 19.052 & 19.104 & 19.174 & 19.216 & 19.256 & 19.289 & 19.308 & 19.303 & 19.263 & 19.183 \\
\hline 10.4 & 19.319 & 19.365 & 19.419 & 19.466 & 19.534 & 19.575 & 19.611 & 19.636 & 19.641 & 19.612 & 19.545 \\
\hline 10.6 & 19.637 & 19.679 & 19.732 & 19.783 & 19.852 & 19.893 & 19.932 & 19.961 & 19.974 & 19.957 & 19.903 \\
\hline 10.8 & 19.952 & 19.993 & 20.045 & 20.098 & 20.169 & 20.211 & 20.251 & 20.284 & 20.303 & 20.297 & 20.255 \\
\hline 11.0 & 20.264 & 20.307 & 20.356 & 20.411 & 20.459 & 20.527 & 20.568 & 20.604 & 20.629 & 20.632 & 20.602 \\
\hline
\end{tabular}

Table 5: $\log \sigma_{1}$ for ${ }^{12} \mathrm{C}$ at $B_{12}=1$

\begin{tabular}{|c|c|c|c|c|c|c|c|c|c|c|c|}
\hline $\log \rho$ & $\log T=-1.0$ & $\log T=-0.8$ & $\log T=-0.6$ & $\log T=-0.4$ & $\log T=-0.2$ & $\log T=0.0$ & $\log T=0.2$ & $\log T=0.4$ & $\log T=0.6$ & $\log T=0.8$ & $\log T=1.0$ \\
\hline 6.0 & 18.633 & 18.633 & 18.633 & 18.633 & 18.629 & 18.629 & 18.629 & 18.629 & 18.629 & 18.629 & 18.629 \\
\hline 6.2 & 18.833 & 18.833 & 18.833 & 18.833 & 18.831 & 18.829 & 18.829 & 18.829 & 18.829 & 18.829 & 18.829 \\
\hline 6.4 & 19.033 & 19.033 & 19.033 & 19.033 & 19.032 & 19.029 & 19.029 & 19.029 & 19.029 & 19.029 & 19.029 \\
\hline 6.6 & 19.233 & 19.233 & 19.233 & 19.233 & 19.233 & 19.229 & 19.229 & 19.229 & 19.229 & 19.229 & 19.229 \\
\hline 6.8 & 19.433 & 19.433 & 19.433 & 19.433 & 19.433 & 19.429 & 19.429 & 19.429 & 19.429 & 19.429 & 19.429 \\
\hline 7.0 & 19.633 & 19.633 & 19.633 & 19.633 & 19.633 & 19.629 & 19.629 & 19.629 & 19.629 & 19.629 & 19.629 \\
\hline 7.2 & 19.833 & 19.833 & 19.833 & 19.833 & 19.833 & 19.831 & 19.829 & 19.829 & 19.829 & 19.829 & 19.829 \\
\hline 7.4 & 20.032 & 20.032 & 20.032 & 20.032 & 20.032 & 20.032 & 20.028 & 20.029 & 20.029 & 20.029 & 20.029 \\
\hline 7.6 & 20.232 & 20.232 & 20.232 & 20.232 & 20.232 & 20.232 & 20.228 & 20.228 & 20.229 & 20.229 & 20.229 \\
\hline 7.8 & 20.431 & 20.431 & 20.431 & 20.430 & 20.430 & 20.431 & 20.429 & 20.428 & 20.428 & 20.429 & 20.429 \\
\hline 8.0 & 20.630 & 20.629 & 20.629 & 20.628 & 20.628 & 20.629 & 20.629 & 20.626 & 20.627 & 20.628 & 20.628 \\
\hline 8.2 & 20.828 & 20.827 & 20.825 & 20.824 & 20.824 & 20.824 & 20.826 & 20.824 & 20.826 & 20.827 & 20.828 \\
\hline 8.4 & 21.024 & 21.022 & 21.020 & 21.018 & 21.017 & 21.017 & 21.019 & 21.020 & 21.022 & 21.025 & 21.027 \\
\hline 8.6 & 21.220 & 21.215 & 21.211 & 21.207 & 21.204 & 21.203 & 21.206 & 21.212 & 21.215 & 21.220 & 21.224 \\
\hline 8.8 & 21.410 & 21.402 & 21.396 & 21.389 & 21.383 & 21.380 & 21.383 & 21.393 & 21.400 & 21.411 & 21.418 \\
\hline 9.0 & 21.595 & 21.582 & 21.571 & 21.560 & 21.549 & 21.542 & 21.544 & 21.557 & 21.573 & 21.592 & 21.606 \\
\hline 9.2 & 21.771 & 21.757 & 21.732 & 21.714 & 21.696 & 21.682 & 21.680 & 21.697 & 21.726 & 21.755 & 21.782 \\
\hline 9.4 & 21.932 & 21.910 & 21.872 & 21.845 & 21.817 & 21.793 & 21.785 & 21.802 & 21.844 & 21.890 & 21.938 \\
\hline 9.6 & 22.074 & 22.040 & 21.984 & 21.946 & 21.906 & 21.871 & 21.853 & 21.867 & 21.919 & 21.987 & 22.060 \\
\hline 9.8 & 22.190 & 22.142 & 22.094 & 22.016 & 21.964 & 21.916 & 21.886 & 21.892 & 21.947 & 22.039 & 22.137 \\
\hline 10.0 & 22.277 & 22.215 & 22.151 & 22.055 & 21.993 & 21.934 & 21.891 & 21.884 & 21.934 & 22.038 & 22.162 \\
\hline 10.2 & 22.332 & 22.261 & 22.182 & 22.069 & 21.999 & 21.931 & 21.877 & 21.855 & 21.891 & 21.995 & 22.137 \\
\hline 10.4 & 22.358 & 22.284 & 22.194 & 22.112 & 21.990 & 21.916 & 21.851 & 21.815 & 21.832 & 21.924 & 22.078 \\
\hline 10.6 & 22.364 & 22.289 & 22.193 & 22.100 & 21.971 & 21.892 & 21.820 & 21.771 & 21.768 & 21.840 & 21.990 \\
\hline 10.8 & 22.358 & 22.281 & 22.184 & 22.082 & 21.945 & 21.863 & 21.786 & 21.726 & 21.705 & 21.753 & 21.887 \\
\hline 11.0 & 22.348 & 22.265 & 22.171 & 22.063 & 21.968 & 21.833 & 21.752 & 21.684 & 21.647 & 21.670 & 21.781 \\
\hline
\end{tabular}

Table 6: $\log \sigma_{1}$ for ${ }^{12} \mathrm{C}$ at $B_{12}=10$

\begin{tabular}{|c|c|c|c|c|c|c|c|c|c|c|c|}
\hline $\log \rho$ & $\log T=-1.0$ & $\log T=-0.8$ & $\log T=-0.6$ & $\log T=-0.4$ & $\log T=-0.2$ & $\log T=0.0$ & $\log T=0.2$ & $\log T=0.4$ & $\log T=0.6$ & $\log T=0.8$ & $\log T=1.0$ \\
\hline 6.0 & 17.633 & 17.633 & 17.633 & 17.633 & 17.629 & 17.629 & 17.629 & 17.629 & 17.629 & 17.629 & 17.629 \\
\hline 6.2 & 17.833 & 17.833 & 17.833 & 17.833 & 17.831 & 17.829 & 17.829 & 17.829 & 17.829 & 17.829 & 17.829 \\
\hline 6.4 & 18.033 & 18.033 & 18.033 & 18.033 & 18.032 & 18.029 & 18.029 & 18.029 & 18.029 & 18.029 & 18.029 \\
\hline 6.6 & 18.233 & 18.233 & 18.233 & 18.233 & 18.233 & 18.229 & 18.229 & 18.229 & 18.229 & 18.229 & 18.229 \\
\hline 6.8 & 18.433 & 18.433 & 18.433 & 18.433 & 18.433 & 18.429 & 18.429 & 18.429 & 18.429 & 18.429 & 18.429 \\
\hline 7.0 & 18.633 & 18.633 & 18.633 & 18.633 & 18.633 & 18.629 & 18.629 & 18.629 & 18.629 & 18.629 & 18.629 \\
\hline 7.2 & 18.833 & 18.833 & 18.833 & 18.833 & 18.833 & 18.832 & 18.829 & 18.829 & 18.829 & 18.829 & 18.829 \\
\hline 7.4 & 19.033 & 19.033 & 19.033 & 19.033 & 19.033 & 19.033 & 19.029 & 19.029 & 19.029 & 19.029 & 19.029 \\
\hline 7.6 & 19.233 & 19.233 & 19.233 & 19.233 & 19.233 & 19.233 & 19.229 & 19.229 & 19.229 & 19.229 & 19.229 \\
\hline 7.8 & 19.433 & 19.433 & 19.433 & 19.433 & 19.433 & 19.433 & 19.431 & 19.429 & 19.429 & 19.429 & 19.429 \\
\hline 8.0 & 19.633 & 19.633 & 19.633 & 19.633 & 19.633 & 19.633 & 19.633 & 19.629 & 19.629 & 19.629 & 19.629 \\
\hline 8.2 & 19.833 & 19.833 & 19.833 & 19.833 & 19.833 & 19.833 & 19.833 & 19.829 & 19.829 & 19.829 & 19.829 \\
\hline 8.4 & 20.033 & 20.033 & 20.033 & 20.033 & 20.033 & 20.033 & 20.033 & 20.030 & 20.029 & 20.029 & 20.029 \\
\hline 8.6 & 20.233 & 20.233 & 20.233 & 20.233 & 20.233 & 20.233 & 20.233 & 20.232 & 20.229 & 20.229 & 20.229 \\
\hline 8.8 & 20.433 & 20.433 & 20.433 & 20.433 & 20.433 & 20.433 & 20.433 & 20.433 & 20.428 & 20.429 & 20.429 \\
\hline 9.0 & 20.633 & 20.633 & 20.633 & 20.632 & 20.632 & 20.632 & 20.632 & 20.632 & 20.629 & 20.628 & 20.629 \\
\hline 9.2 & 20.833 & 20.833 & 20.832 & 20.832 & 20.832 & 20.832 & 20.831 & 20.832 & 20.831 & 20.828 & 20.828 \\
\hline 9.4 & 21.032 & 21.032 & 21.031 & 21.031 & 21.031 & 21.030 & 21.030 & 21.030 & 21.030 & 21.027 & 21.028 \\
\hline 9.6 & 21.231 & 21.231 & 21.230 & 21.229 & 21.228 & 21.228 & 21.227 & 21.227 & 21.228 & 21.226 & 21.226 \\
\hline 9.8 & 21.430 & 21.429 & 21.428 & 21.426 & 21.425 & 21.423 & 21.422 & 21.422 & 21.423 & 21.424 & 21.423 \\
\hline 10.0 & 21.628 & 21.626 & 21.625 & 21.621 & 21.619 & 21.616 & 21.614 & 21.612 & 21.613 & 21.617 & 21.617 \\
\hline 10.2 & 21.824 & 21.822 & 21.819 & 21.813 & 21.809 & 21.804 & 21.799 & 21.796 & 21.796 & 21.801 & 21.806 \\
\hline 10.4 & 22.017 & 22.014 & 22.008 & 22.003 & 21.992 & 21.984 & 21.975 & 21.968 & 21.966 & 21.973 & 21.986 \\
\hline 10.6 & 22.206 & 22.201 & 22.192 & 22.182 & 22.164 & 22.151 & 22.136 & 22.124 & 22.118 & 22.125 & 22.145 \\
\hline 10.8 & 22.388 & 22.380 & 22.366 & 22.349 & 22.320 & 22.299 & 22.276 & 22.255 & 22.243 & 22.248 & 22.276 \\
\hline 11.0 & 22.560 & 22.546 & 22.526 & 22.499 & 22.471 & 22.423 & 22.390 & 22.358 & 22.335 & 22.336 & 22.370 \\
\hline
\end{tabular}


Table 7: $\log \sigma_{1}$ for ${ }^{12} \mathrm{C}$ at $B_{12}=100$

\begin{tabular}{|c|c|c|c|c|c|c|c|c|c|c|c|}
\hline $\log \rho$ & $\log T=-1.0$ & $\log T=-0.8$ & $\log T=-0.6$ & $\log T=-0.4$ & $\log T=-0.2$ & $\log T=0.0$ & $\log T=0.2$ & $\log T=0.4$ & $\log T=0.6$ & $\log T=0.8$ & $\log T=1.0$ \\
\hline 6.0 & 16.633 & 16.633 & 16.633 & 16.633 & 16.629 & 16.629 & 16.629 & 16.629 & 16.629 & 16.629 & 16.629 \\
\hline 6.2 & 16.833 & 16.833 & 16.833 & 16.833 & 16.831 & 16.829 & 16.829 & 16.829 & 16.829 & 16.829 & 16.829 \\
\hline 6.4 & 17.033 & 17.033 & 17.033 & 17.033 & 17.032 & 17.029 & 17.029 & 17.029 & 17.029 & 17.029 & 17.029 \\
\hline 6.6 & 17.233 & 17.233 & 17.233 & 17.233 & 17.233 & 17.229 & 17.229 & 17.229 & 17.229 & 17.229 & 17.229 \\
\hline 6.8 & 17.433 & 17.433 & 17.433 & 17.433 & 17.433 & 17.429 & 17.429 & 17.429 & 17.429 & 17.429 & 17.429 \\
\hline 7.0 & 17.633 & 17.633 & 17.633 & 17.633 & 17.633 & 17.629 & 17.629 & 17.629 & 17.629 & 17.629 & 17.629 \\
\hline 7.2 & 17.833 & 17.833 & 17.833 & 17.833 & 17.833 & 17.832 & 17.829 & 17.829 & 17.829 & 17.829 & 17.829 \\
\hline 7.4 & 18.033 & 18.033 & 18.033 & 18.033 & 18.033 & 18.033 & 18.029 & 18.029 & 18.029 & 18.029 & 18.029 \\
\hline 7.6 & 18.233 & 18.233 & 18.233 & 18.233 & 18.233 & 18.233 & 18.229 & 18.229 & 18.229 & 18.229 & 18.229 \\
\hline 7.8 & 18.433 & 18.433 & 18.433 & 18.433 & 18.433 & 18.433 & 18.431 & 18.429 & 18.429 & 18.429 & 18.429 \\
\hline 8.0 & 18.633 & 18.633 & 18.633 & 18.633 & 18.633 & 18.633 & 18.633 & 18.629 & 18.629 & 18.629 & 18.629 \\
\hline 8.2 & 18.833 & 18.833 & 18.833 & 18.833 & 18.833 & 18.833 & 18.833 & 18.829 & 18.829 & 18.829 & 18.829 \\
\hline 8.4 & 19.033 & 19.033 & 19.033 & 19.033 & 19.033 & 19.033 & 19.033 & 19.030 & 19.029 & 19.029 & 19.029 \\
\hline 8.6 & 19.233 & 19.233 & 19.233 & 19.233 & 19.233 & 19.233 & 19.233 & 19.232 & 19.229 & 19.229 & 19.229 \\
\hline 8.8 & 19.433 & 19.433 & 19.433 & 19.433 & 19.433 & 19.433 & 19.433 & 19.433 & 19.429 & 19.429 & 19.429 \\
\hline 9.0 & 19.633 & 19.633 & 19.633 & 19.633 & 19.633 & 19.633 & 19.633 & 19.633 & 19.630 & 19.629 & 19.629 \\
\hline 9.2 & 19.833 & 19.833 & 19.833 & 19.833 & 19.833 & 19.833 & 19.833 & 19.833 & 19.832 & 19.829 & 19.829 \\
\hline 9.4 & 20.033 & 20.033 & 20.033 & 20.033 & 20.033 & 20.033 & 20.033 & 20.033 & 20.033 & 20.029 & 20.029 \\
\hline 9.6 & 20.233 & 20.233 & 20.233 & 20.233 & 20.233 & 20.233 & 20.233 & 20.233 & 20.233 & 20.230 & 20.229 \\
\hline 9.8 & 20.433 & 20.433 & 20.433 & 20.433 & 20.433 & 20.433 & 20.433 & 20.433 & 20.433 & 20.432 & 20.429 \\
\hline 10.0 & 20.633 & 20.633 & 20.633 & 20.633 & 20.633 & 20.633 & 20.633 & 20.633 & 20.633 & 20.633 & 20.629 \\
\hline 10.2 & 20.833 & 20.833 & 20.833 & 20.833 & 20.833 & 20.833 & 20.833 & 20.833 & 20.833 & 20.833 & 20.829 \\
\hline 10.4 & 21.033 & 21.033 & 21.033 & 21.033 & 21.033 & 21.033 & 21.033 & 21.033 & 21.033 & 21.033 & 21.032 \\
\hline 10.6 & 21.233 & 21.233 & 21.233 & 21.233 & 21.233 & 21.232 & 21.232 & 21.232 & 21.232 & 21.232 & 21.232 \\
\hline 10.8 & 21.433 & 21.433 & 21.433 & 21.432 & 21.432 & 21.432 & 21.431 & 21.431 & 21.431 & 21.431 & 21.431 \\
\hline 11.0 & 21.633 & 21.632 & 21.632 & 21.632 & 21.631 & 21.631 & 21.630 & 21.630 & 21.629 & 21.629 & 21.629 \\
\hline
\end{tabular}

Table 8: $\log \sigma$ for ${ }^{56} \mathrm{Fe}$

\begin{tabular}{|c|c|c|c|c|c|c|c|c|c|c|c|}
\hline $\log \rho$ & $\log T=-1.0$ & $\log T=-0.8$ & $\log T=-0.6$ & $\log T=-0.4$ & $\log T=-0.2$ & $\log T=0.0$ & $\log T=0.2$ & $\log T=0.4$ & $\log T=0.6$ & $\log T=0.8$ & $\log T=1.0$ \\
\hline 6.0 & 20.144 & 20.260 & 20.375 & 20.506 & 20.628 & 20.762 & 20.902 & 21.048 & 21.200 & 21.357 & 21.522 \\
\hline 6.2 & 20.213 & 20.310 & 20.413 & 20.537 & 20.658 & 20.785 & 20.923 & 21.066 & 21.216 & 21.372 & 21.536 \\
\hline 6.4 & 20.293 & 20.368 & 20.456 & 20.570 & 20.689 & 20.809 & 20.944 & 21.085 & 21.233 & 21.388 & 21.550 \\
\hline 6.6 & 20.382 & 20.435 & 20.517 & 20.607 & 20.720 & 20.834 & 20.966 & 21.104 & 21.250 & 21.403 & 21.565 \\
\hline 6.8 & 20.477 & 20.510 & 20.572 & 20.647 & 20.753 & 20.861 & 20.989 & 21.125 & 21.268 & 21.420 & 21.580 \\
\hline 7.0 & 20.576 & 20.592 & 20.634 & 20.692 & 20.788 & 20.889 & 21.013 & 21.146 & 21.287 & 21.437 & 21.595 \\
\hline 7.2 & 20.675 & 20.680 & 20.702 & 20.756 & 20.826 & 20.925 & 21.038 & 21.168 & 21.307 & 21.454 & 21.611 \\
\hline 7.4 & 20.772 & 20.769 & 20.777 & 20.813 & 20.867 & 20.961 & 21.065 & 21.191 & 21.327 & 21.473 & 21.628 \\
\hline 7.6 & 20.865 & 20.858 & 20.856 & 20.875 & 20.913 & 20.997 & 21.094 & 21.215 & 21.348 & 21.492 & 21.645 \\
\hline 7.8 & 20.954 & 20.944 & 20.937 & 20.943 & 20.980 & 21.036 & 21.127 & 21.241 & 21.370 & 21.511 & 21.663 \\
\hline 8.0 & 21.040 & 21.028 & 21.019 & 21.015 & 21.036 & 21.079 & 21.165 & 21.268 & 21.394 & 21.532 & 21.682 \\
\hline 8.2 & 21.123 & 21.110 & 21.099 & 21.090 & 21.098 & 21.125 & 21.202 & 21.297 & 21.418 & 21.553 & 21.701 \\
\hline 8.4 & 21.203 & 21.189 & 21.177 & 21.166 & 21.165 & 21.192 & 21.242 & 21.329 & 21.444 & 21.576 & 21.721 \\
\hline 8.6 & 21.280 & 21.267 & 21.254 & 21.242 & 21.234 & 21.249 & 21.285 & 21.368 & 21.471 & 21.599 & 21.742 \\
\hline 8.8 & 21.356 & 21.343 & 21.329 & 21.318 & 21.306 & 21.310 & 21.331 & 21.406 & 21.500 & 21.624 & 21.764 \\
\hline 9.0 & 21.429 & 21.418 & 21.404 & 21.392 & 21.379 & 21.375 & 21.399 & 21.446 & 21.532 & 21.650 & 21.787 \\
\hline 9.2 & 21.502 & 21.492 & 21.479 & 21.465 & 21.453 & 21.443 & 21.455 & 21.489 & 21.572 & 21.678 & 21.811 \\
\hline 9.4 & 21.574 & 21.565 & 21.552 & 21.538 & 21.526 & 21.514 & 21.516 & 21.536 & 21.610 & 21.707 & 21.837 \\
\hline 9.6 & 21.645 & 21.637 & 21.625 & 21.611 & 21.599 & 21.586 & 21.580 & 21.603 & 21.651 & 21.739 & 21.864 \\
\hline 9.8 & 21.716 & 21.708 & 21.698 & 21.684 & 21.671 & 21.658 & 21.648 & 21.660 & 21.694 & 21.780 & 21.892 \\
\hline 10.0 & 21.787 & 21.779 & 21.770 & 21.758 & 21.743 & 21.731 & 21.719 & 21.721 & 21.742 & 21.819 & 21.922 \\
\hline 10.2 & 21.858 & 21.851 & 21.842 & 21.831 & 21.816 & 21.804 & 21.791 & 21.786 & 21.810 & 21.860 & 21.955 \\
\hline 10.4 & 21.929 & 21.922 & 21.914 & 21.904 & 21.890 & 21.876 & 21.864 & 21.854 & 21.867 & 21.905 & 21.998 \\
\hline 10.6 & 22.001 & 21.994 & 21.986 & 21.977 & 21.963 & 21.949 & 21.937 & 21.925 & 21.929 & 21.953 & 22.038 \\
\hline 10.8 & 22.073 & 22.066 & 22.058 & 22.050 & 22.038 & 22.023 & 22.011 & 21.998 & 21.995 & 22.023 & 22.081 \\
\hline 11.0 & 22.143 & 22.140 & 22.132 & 22.123 & 22.113 & 22.099 & 22.085 & 22.073 & 22.065 & 22.082 & 22.127 \\
\hline
\end{tabular}

Table 9: $\log \sigma_{0}$ for ${ }^{56} \mathrm{Fe}$ at $B_{12}=1$

\begin{tabular}{|c|c|c|c|c|c|c|c|c|c|c|c|}
\hline $\log \rho$ & $\log T=-1.0$ & $\log T=-0.8$ & $\log T=-0.6$ & $\log T=-0.4$ & $\log T=-0.2$ & $\log T=0.0$ & $\log T=0.2$ & $\log T=0.4$ & $\log T=0.6$ & $\log T=0.8$ & $\log T=1.0$ \\
\hline 6.0 & 17.169 & 17.065 & 16.945 & 16.800 & 16.654 & 16.512 & 16.370 & 16.226 & 16.078 & 15.925 & 15.766 \\
\hline 6.2 & 17.472 & 17.395 & 17.295 & 17.161 & 17.023 & 16.886 & 16.748 & 16.607 & 16.460 & 16.309 & 16.151 \\
\hline 6.4 & 17.768 & 17.715 & 17.637 & 17.519 & 17.391 & 17.258 & 17.124 & 16.986 & 16.842 & 16.692 & 16.536 \\
\hline 6.6 & 18.057 & 18.026 & 17.957 & 17.872 & 17.755 & 17.629 & 17.499 & 17.364 & 17.223 & 17.075 & 16.920 \\
\hline 6.8 & 18.345 & 18.331 & 18.285 & 18.219 & 18.115 & 17.997 & 17.873 & 17.742 & 17.603 & 17.457 & 17.304 \\
\hline 7.0 & 18.634 & 18.631 & 18.605 & 18.560 & 18.471 & 18.362 & 18.245 & 18.117 & 17.982 & 17.839 & 17.687 \\
\hline 7.2 & 18.926 & 18.930 & 18.919 & 18.879 & 18.821 & 18.725 & 18.614 & 18.492 & 18.360 & 18.219 & 18.070 \\
\hline 7.4 & 19.221 & 19.228 & 19.228 & 19.205 & 19.165 & 19.082 & 18.979 & 18.863 & 18.736 & 18.598 & 18.452 \\
\hline 7.6 & 19.519 & 19.527 & 19.533 & 19.524 & 19.500 & 19.432 & 19.339 & 19.231 & 19.109 & 18.976 & 18.832 \\
\hline 7.8 & 19.816 & 19.825 & 19.833 & 19.833 & 19.809 & 19.771 & 19.692 & 19.593 & 19.479 & 19.351 & 19.210 \\
\hline 8.0 & 20.108 & 20.118 & 20.126 & 20.130 & 20.119 & 20.093 & 20.031 & 19.944 & 19.840 & 19.720 & 19.586 \\
\hline 8.2 & 20.391 & 20.399 & 20.405 & 20.411 & 20.407 & 20.394 & 20.348 & 20.279 & 20.189 & 20.081 & 19.955 \\
\hline 8.4 & 20.655 & 20.661 & 20.665 & 20.669 & 20.669 & 20.657 & 20.635 & 20.587 & 20.518 & 20.426 & 20.314 \\
\hline 8.6 & 20.893 & 20.895 & 20.897 & 20.898 & 20.897 & 20.892 & 20.882 & 20.858 & 20.814 & 20.747 & 20.655 \\
\hline 8.8 & 21.098 & 21.096 & 21.094 & 21.092 & 21.088 & 21.086 & 21.083 & 21.082 & 21.066 & 21.031 & 20.968 \\
\hline 9.0 & 21.267 & 21.263 & 21.257 & 21.251 & 21.245 & 21.241 & 21.246 & 21.256 & 21.268 & 21.268 & 21.241 \\
\hline 9.2 & 21.404 & 21.398 & 21.390 & 21.381 & 21.373 & 21.365 & 21.370 & 21.385 & 21.421 & 21.452 & 21.465 \\
\hline 9.4 & 21.517 & 21.510 & 21.501 & 21.489 & 21.480 & 21.469 & 21.469 & 21.481 & 21.531 & 21.584 & 21.635 \\
\hline 9.6 & 21.613 & 21.606 & 21.596 & 21.584 & 21.572 & 21.561 & 21.555 & 21.574 & 21.610 & 21.676 & 21.755 \\
\hline 9.8 & 21.698 & 21.691 & 21.681 & 21.669 & 21.656 & 21.644 & 21.635 & 21.645 & 21.674 & 21.748 & 21.838 \\
\hline 10.0 & 21.777 & 21.769 & 21.761 & 21.749 & 21.735 & 21.723 & 21.711 & 21.713 & 21.732 & 21.804 & 21.896 \\
\hline 10.2 & 21.853 & 21.845 & 21.837 & 21.826 & 21.812 & 21.799 & 21.786 & 21.781 & 21.805 & 21.853 & 21.943 \\
\hline 10.4 & 21.926 & 21.919 & 21.911 & 21.901 & 21.887 & 21.873 & 21.861 & 21.852 & 21.864 & 21.901 & 21.992 \\
\hline 10.6 & 21.999 & 21.992 & 21.984 & 21.975 & 21.962 & 21.948 & 21.936 & 21.924 & 21.927 & 21.951 & 22.036 \\
\hline 10.8 & 22.072 & 22.066 & 22.057 & 22.049 & 22.037 & 22.023 & 22.010 & 21.998 & 21.994 & 22.022 & 22.080 \\
\hline 11.0 & 22.142 & 22.139 & 22.132 & 22.123 & 22.112 & 22.098 & 22.085 & 22.073 & 22.064 & 22.081 & 22.127 \\
\hline
\end{tabular}


Table 10: $\log \sigma_{0}$ for ${ }^{56} \mathrm{Fe}$ at $B_{12}=10$

\begin{tabular}{|c|c|c|c|c|c|c|c|c|c|c|c|}
\hline $\log \rho$ & $\log T=-1.0$ & $\log T=-0.8$ & $\log T=-0.6$ & $\log T=-0.4$ & $\log T=-0.2$ & $\log T=0.0$ & $\log T=0.2$ & $\log T=0.4$ & $\log T=0.6$ & $\log T=0.8$ & $\log T=1.0$ \\
\hline 6.0 & 15.171 & 15.068 & 14.948 & 14.801 & 14.655 & 14.512 & 14.370 & 14.226 & 14.078 & 13.925 & 13.766 \\
\hline 6.2 & 15.475 & 15.398 & 15.298 & 15.163 & 15.024 & 14.886 & 14.748 & 14.607 & 14.460 & 14.309 & 14.151 \\
\hline 6.4 & 15.771 & 15.718 & 15.641 & 15.522 & 15.392 & 15.259 & 15.125 & 14.986 & 14.842 & 14.692 & 14.536 \\
\hline 6.6 & 16.061 & 16.031 & 15.961 & 15.875 & 15.757 & 15.630 & 15.500 & 15.365 & 15.223 & 15.075 & 14.920 \\
\hline 6.8 & 16.350 & 16.336 & 16.290 & 16.224 & 16.118 & 15.999 & 15.874 & 15.742 & 15.603 & 15.457 & 15.304 \\
\hline 7.0 & 16.640 & 16.638 & 16.612 & 16.568 & 16.476 & 16.366 & 16.246 & 16.119 & 15.983 & 15.839 & 15.688 \\
\hline 7.2 & 16.934 & 16.939 & 16.929 & 16.889 & 16.830 & 16.731 & 16.617 & 16.494 & 16.361 & 16.220 & 16.070 \\
\hline 7.4 & 17.234 & 17.242 & 17.243 & 17.220 & 17.180 & 17.093 & 16.986 & 16.868 & 16.739 & 16.600 & 16.452 \\
\hline 7.6 & 17.539 & 17.549 & 17.556 & 17.548 & 17.524 & 17.452 & 17.353 & 17.240 & 17.115 & 16.979 & 16.834 \\
\hline 7.8 & 17.848 & 17.859 & 17.870 & 17.872 & 17.848 & 17.806 & 17.718 & 17.611 & 17.490 & 17.357 & 17.214 \\
\hline 8.0 & 18.162 & 18.174 & 18.186 & 18.194 & 18.183 & 18.156 & 18.080 & 17.980 & 17.864 & 17.735 & 17.594 \\
\hline 8.2 & 18.479 & 18.492 & 18.504 & 18.516 & 18.515 & 18.502 & 18.438 & 18.346 & 18.236 & 18.111 & 17.973 \\
\hline 8.4 & 18.798 & 18.812 & 18.824 & 18.837 & 18.843 & 18.826 & 18.792 & 18.710 & 18.606 & 18.485 & 18.351 \\
\hline 8.6 & 19.119 & 19.132 & 19.145 & 19.158 & 19.169 & 19.163 & 19.141 & 19.071 & 18.974 & 18.858 & 18.727 \\
\hline 8.8 & 19.441 & 19.454 & 19.467 & 19.479 & 19.492 & 19.494 & 19.485 & 19.427 & 19.338 & 19.229 & 19.102 \\
\hline 9.0 & 19.763 & 19.774 & 19.787 & 19.799 & 19.813 & 19.820 & 19.806 & 19.776 & 19.697 & 19.596 & 19.474 \\
\hline 9.2 & 20.084 & 20.092 & 20.105 & 20.117 & 20.129 & 20.140 & 20.135 & 20.116 & 20.051 & 19.957 & 19.843 \\
\hline 9.4 & 20.399 & 20.406 & 20.417 & 20.429 & 20.440 & 20.451 & 20.453 & 20.444 & 20.392 & 20.309 & 20.204 \\
\hline 9.6 & 20.704 & 20.711 & 20.719 & 20.730 & 20.739 & 20.749 & 20.754 & 20.742 & 20.715 & 20.647 & 20.555 \\
\hline 9.8 & 20.995 & 21.000 & 21.006 & 21.014 & 21.021 & 21.028 & 21.034 & 21.028 & 21.012 & 20.963 & 20.887 \\
\hline 10.0 & 21.261 & 21.265 & 21.268 & 21.273 & 21.277 & 21.281 & 21.284 & 21.282 & 21.273 & 21.244 & 21.190 \\
\hline 10.2 & 21.496 & 21.497 & 21.498 & 21.499 & 21.500 & 21.500 & 21.499 & 21.497 & 21.493 & 21.482 & 21.455 \\
\hline 10.4 & 21.694 & 21.693 & 21.691 & 21.689 & 21.686 & 21.682 & 21.678 & 21.673 & 21.673 & 21.673 & 21.675 \\
\hline 10.6 & 21.855 & 21.853 & 21.849 & 21.844 & 21.838 & 21.831 & 21.824 & 21.816 & 21.815 & 21.820 & 21.846 \\
\hline 10.8 & 21.986 & 21.982 & 21.977 & 21.971 & 21.963 & 21.953 & 21.944 & 21.934 & 21.930 & 21.945 & 21.973 \\
\hline 11.0 & 22.091 & 22.091 & 22.085 & 22.078 & 22.069 & 22.058 & 22.046 & 22.036 & 22.028 & 22.040 & 22.070 \\
\hline
\end{tabular}

Table 11: $\log \sigma_{0}$ for ${ }^{56} \mathrm{Fe}$ at $B_{12}=100$

\begin{tabular}{|c|c|c|c|c|c|c|c|c|c|c|c|}
\hline $\log \rho$ & $\log T=-1.0$ & $\log T=-0.8$ & $\log T=-0.6$ & $\log T=-0.4$ & $\log T=-0.2$ & $\log T=0.0$ & $\log T=0.2$ & $\log T=0.4$ & $\log T=0.6$ & $\log T=0.8$ & $\log T=1.0$ \\
\hline 6.0 & 13.171 & 13.068 & 12.948 & 12.801 & 12.655 & 12.512 & 12.370 & 12.226 & 12.078 & 11.925 & 11.766 \\
\hline 6.2 & 13.475 & 13.398 & 13.298 & 13.163 & 13.025 & 12.886 & 12.748 & 12.607 & 12.460 & 12.309 & 12.151 \\
\hline 6.4 & 13.771 & 13.718 & 13.641 & 13.522 & 13.392 & 13.259 & 13.125 & 12.986 & 12.842 & 12.692 & 12.536 \\
\hline 6.6 & 14.061 & 14.031 & 13.961 & 13.876 & 13.757 & 13.630 & 13.500 & 13.365 & 13.223 & 13.075 & 12.920 \\
\hline 6.8 & 14.350 & 14.336 & 14.290 & 14.224 & 14.118 & 13.999 & 13.874 & 13.742 & 13.603 & 13.457 & 13.304 \\
\hline 7.0 & 14.640 & 14.638 & 14.612 & 14.568 & 14.476 & 14.366 & 14.246 & 14.119 & 13.983 & 13.839 & 13.688 \\
\hline 7.2 & 14.934 & 14.939 & 14.929 & 14.889 & 14.830 & 14.731 & 14.617 & 14.494 & 14.361 & 14.220 & 14.070 \\
\hline 7.4 & 15.234 & 15.242 & 15.243 & 15.221 & 15.180 & 15.093 & 14.986 & 14.868 & 14.739 & 14.600 & 14.452 \\
\hline 7.6 & 15.539 & 15.549 & 15.557 & 15.548 & 15.525 & 15.452 & 15.353 & 15.240 & 15.115 & 14.979 & 14.834 \\
\hline 7.8 & 15.849 & 15.860 & 15.871 & 15.872 & 15.848 & 15.806 & 15.718 & 15.611 & 15.490 & 15.358 & 15.214 \\
\hline 8.0 & 16.163 & 16.175 & 16.186 & 16.195 & 16.184 & 16.157 & 16.081 & 15.980 & 15.864 & 15.735 & 15.594 \\
\hline 8.2 & 16.480 & 16.493 & 16.505 & 16.517 & 16.516 & 16.503 & 16.440 & 16.347 & 16.236 & 16.111 & 15.973 \\
\hline 8.4 & 16.800 & 16.813 & 16.826 & 16.839 & 16.845 & 16.828 & 16.794 & 16.712 & 16.607 & 16.486 & 16.351 \\
\hline 8.6 & 17.122 & 17.135 & 17.149 & 17.161 & 17.173 & 17.166 & 17.145 & 17.074 & 16.976 & 16.860 & 16.728 \\
\hline 8.8 & 17.447 & 17.459 & 17.473 & 17.485 & 17.499 & 17.501 & 17.493 & 17.433 & 17.343 & 17.232 & 17.104 \\
\hline 9.0 & 17.773 & 17.784 & 17.798 & 17.811 & 17.825 & 17.833 & 17.819 & 17.788 & 17.707 & 17.603 & 17.479 \\
\hline 9.2 & 18.100 & 18.110 & 18.124 & 18.137 & 18.150 & 18.163 & 18.158 & 18.139 & 18.070 & 17.971 & 17.852 \\
\hline 9.4 & 18.428 & 18.437 & 18.450 & 18.464 & 18.476 & 18.491 & 18.494 & 18.487 & 18.428 & 18.337 & 18.224 \\
\hline 9.6 & 18.757 & 18.765 & 18.776 & 18.790 & 18.803 & 18.817 & 18.826 & 18.813 & 18.783 & 18.701 & 18.593 \\
\hline 9.8 & 19.085 & 19.093 & 19.103 & 19.117 & 19.130 & 19.144 & 19.156 & 19.152 & 19.133 & 19.063 & 18.961 \\
\hline 10.0 & 19.413 & 19.421 & 19.430 & 19.443 & 19.457 & 19.469 & 19.484 & 19.487 & 19.479 & 19.420 & 19.326 \\
\hline 10.2 & 19.741 & 19.748 & 19.757 & 19.768 & 19.782 & 19.795 & 19.809 & 19.817 & 19.803 & 19.772 & 19.687 \\
\hline 10.4 & 20.067 & 20.074 & 20.082 & 20.092 & 20.105 & 20.119 & 20.132 & 20.143 & 20.138 & 20.118 & 20.044 \\
\hline 10.6 & 20.391 & 20.397 & 20.405 & 20.414 & 20.426 & 20.440 & 20.451 & 20.464 & 20.466 & 20.456 & 20.394 \\
\hline 10.8 & 20.710 & 20.716 & 20.723 & 20.731 & 20.742 & 20.755 & 20.766 & 20.779 & 20.785 & 20.769 & 20.734 \\
\hline 11.0 & 21.007 & 21.028 & 21.034 & 21.042 & 21.050 & 21.062 & 21.073 & 21.083 & 21.092 & 21.084 & 21.059 \\
\hline
\end{tabular}

Table 12: $\log \sigma_{1}$ for ${ }^{56} \mathrm{Fe}$ at $B_{12}=1$

\begin{tabular}{|c|c|c|c|c|c|c|c|c|c|c|c|}
\hline $\log \rho$ & $\log T=-1.0$ & $\log T=-0.8$ & $\log T=-0.6$ & $\log T=-0.4$ & $\log T=-0.2$ & $\log T=0.0$ & $\log T=0.2$ & $\log T=0.4$ & $\log T=0.6$ & $\log T=0.8$ & $\log T=1.0$ \\
\hline 6.0 & 18.600 & 18.601 & 18.601 & 18.601 & 18.597 & 18.596 & 18.597 & 18.597 & 18.597 & 18.597 & 18.597 \\
\hline 6.2 & 18.800 & 18.800 & 18.801 & 18.801 & 18.798 & 18.796 & 18.797 & 18.797 & 18.797 & 18.797 & 18.797 \\
\hline 6.4 & 18.999 & 19.000 & 19.000 & 19.000 & 18.999 & 18.996 & 18.996 & 18.997 & 18.997 & 18.997 & 18.997 \\
\hline 6.6 & 19.199 & 19.199 & 19.199 & 19.200 & 19.200 & 19.196 & 19.196 & 19.197 & 19.197 & 19.197 & 19.197 \\
\hline 6.8 & 19.398 & 19.398 & 19.398 & 19.399 & 19.399 & 19.396 & 19.396 & 19.396 & 19.397 & 19.397 & 19.397 \\
\hline 7.0 & 19.596 & 19.596 & 19.596 & 19.597 & 19.598 & 19.595 & 19.596 & 19.596 & 19.596 & 19.597 & 19.597 \\
\hline 7.2 & 19.793 & 19.793 & 19.793 & 19.794 & 19.795 & 19.796 & 19.794 & 19.795 & 19.796 & 19.796 & 19.796 \\
\hline 7.4 & 19.988 & 19.988 & 19.988 & 19.989 & 19.990 & 19.993 & 19.992 & 19.994 & 19.995 & 19.996 & 19.996 \\
\hline 7.6 & 20.181 & 20.180 & 20.179 & 20.179 & 20.181 & 20.186 & 20.186 & 20.190 & 20.193 & 20.195 & 20.196 \\
\hline 7.8 & 20.368 & 20.366 & 20.365 & 20.364 & 20.367 & 20.372 & 20.378 & 20.384 & 20.389 & 20.392 & 20.394 \\
\hline 8.0 & 20.547 & 20.544 & 20.541 & 20.539 & 20.541 & 20.547 & 20.560 & 20.570 & 20.580 & 20.587 & 20.591 \\
\hline 8.2 & 20.712 & 20.707 & 20.702 & 20.697 & 20.697 & 20.702 & 20.724 & 20.742 & 20.762 & 20.776 & 20.785 \\
\hline 8.4 & 20.856 & 20.848 & 20.841 & 20.833 & 20.829 & 20.841 & 20.861 & 20.893 & 20.927 & 20.954 & 20.972 \\
\hline 8.6 & 20.972 & 20.961 & 20.949 & 20.939 & 20.929 & 20.938 & 20.961 & 21.014 & 21.065 & 21.111 & 21.145 \\
\hline 8.8 & 21.052 & 21.038 & 21.022 & 21.008 & 20.994 & 20.996 & 21.016 & 21.087 & 21.162 & 21.237 & 21.296 \\
\hline 9.0 & 21.095 & 21.080 & 21.060 & 21.042 & 21.024 & 21.017 & 21.050 & 21.110 & 21.210 & 21.319 & 21.412 \\
\hline 9.2 & 21.105 & 21.089 & 21.067 & 21.045 & 21.025 & 21.010 & 21.031 & 21.088 & 21.211 & 21.347 & 21.481 \\
\hline 9.4 & 21.089 & 21.074 & 21.052 & 21.027 & 21.005 & 20.984 & 20.991 & 21.033 & 21.165 & 21.321 & 21.495 \\
\hline 9.6 & 21.057 & 21.041 & 21.020 & 20.994 & 20.970 & 20.947 & 20.940 & 20.989 & 21.087 & 21.252 & 21.457 \\
\hline 9.8 & 21.014 & 20.997 & 20.979 & 20.952 & 20.926 & 20.903 & 20.885 & 20.914 & 20.993 & 21.166 & 21.376 \\
\hline 10.0 & 20.964 & 20.947 & 20.930 & 20.905 & 20.877 & 20.854 & 20.831 & 20.841 & 20.894 & 21.060 & 21.269 \\
\hline 10.2 & 20.910 & 20.895 & 20.878 & 20.855 & 20.827 & 20.802 & 20.778 & 20.772 & 20.830 & 20.947 & 21.148 \\
\hline 10.4 & 20.854 & 20.840 & 20.823 & 20.803 & 20.776 & 20.749 & 20.725 & 20.709 & 20.743 & 20.836 & 21.038 \\
\hline 10.6 & 20.798 & 20.785 & 20.768 & 20.750 & 20.724 & 20.696 & 20.673 & 20.651 & 20.665 & 20.730 & 20.920 \\
\hline 10.8 & 20.744 & 20.731 & 20.715 & 20.697 & 20.674 & 20.645 & 20.620 & 20.597 & 20.595 & 20.664 & 20.803 \\
\hline 11.0 & 20.693 & 20.678 & 20.663 & 20.645 & 20.624 & 20.596 & 20.569 & 20.546 & 20.534 & 20.579 & 20.692 \\
\hline
\end{tabular}


Table 13: $\log \sigma_{1}$ for ${ }^{56} \mathrm{Fe}$ at $B_{12}=10$

\begin{tabular}{|c|c|c|c|c|c|c|c|c|c|c|c|}
\hline $\log \rho$ & $\log T=-1.0$ & $\log T=-0.8$ & $\log T=-0.6$ & $\log T=-0.4$ & $\log T=-0.2$ & $\log T=0.0$ & $\log T=0.2$ & $\log T=0.4$ & $\log T=0.6$ & $\log T=0.8$ & $\log T=1.0$ \\
\hline 6.0 & 17.601 & 17.601 & 17.601 & 17.601 & 17.597 & 17.596 & 17.597 & 17.597 & 17.597 & 17.597 & 17.597 \\
\hline 6.2 & 17.801 & 17.801 & 17.801 & 17.801 & 17.799 & 17.796 & 17.797 & 17.797 & 17.797 & 17.797 & 17.797 \\
\hline 6.4 & 18.001 & 18.001 & 18.001 & 18.001 & 18.000 & 17.996 & 17.997 & 17.997 & 17.997 & 17.997 & 17.997 \\
\hline 6.6 & 18.201 & 18.201 & 18.201 & 18.201 & 18.201 & 18.196 & 18.197 & 18.197 & 18.197 & 18.197 & 18.197 \\
\hline 6.8 & 18.401 & 18.401 & 18.401 & 18.401 & 18.401 & 18.396 & 18.397 & 18.397 & 18.397 & 18.397 & 18.397 \\
\hline 7.0 & 18.601 & 18.601 & 18.601 & 18.601 & 18.601 & 18.596 & 18.597 & 18.597 & 18.597 & 18.597 & 18.597 \\
\hline 7.2 & 18.801 & 18.801 & 18.801 & 18.801 & 18.801 & 18.799 & 18.797 & 18.797 & 18.797 & 18.797 & 18.797 \\
\hline 7.4 & 19.001 & 19.001 & 19.001 & 19.001 & 19.001 & 19.001 & 18.997 & 18.997 & 18.997 & 18.997 & 18.997 \\
\hline 7.6 & 19.201 & 19.201 & 19.201 & 19.201 & 19.201 & 19.201 & 19.196 & 19.197 & 19.197 & 19.197 & 19.197 \\
\hline 7.8 & 19.401 & 19.401 & 19.401 & 19.401 & 19.401 & 19.401 & 19.398 & 19.396 & 19.397 & 19.397 & 19.397 \\
\hline 8.0 & 19.601 & 19.600 & 19.601 & 19.601 & 19.601 & 19.601 & 19.600 & 19.596 & 19.596 & 19.597 & 19.597 \\
\hline 8.2 & 19.800 & 19.800 & 19.800 & 19.800 & 19.800 & 19.800 & 19.800 & 19.796 & 19.796 & 19.796 & 19.797 \\
\hline 8.4 & 19.999 & 19.999 & 19.999 & 19.999 & 19.999 & 19.999 & 19.999 & 19.996 & 19.996 & 19.996 & 19.996 \\
\hline 8.6 & 20.198 & 20.198 & 20.198 & 20.198 & 20.197 & 20.197 & 20.198 & 20.197 & 20.195 & 20.196 & 20.196 \\
\hline 8.8 & 20.396 & 20.396 & 20.395 & 20.395 & 20.394 & 20.394 & 20.394 & 20.396 & 20.393 & 20.394 & 20.395 \\
\hline 9.0 & 20.592 & 20.591 & 20.591 & 20.590 & 20.589 & 20.589 & 20.589 & 20.590 & 20.589 & 20.592 & 20.594 \\
\hline 9.2 & 20.784 & 20.784 & 20.782 & 20.781 & 20.780 & 20.779 & 20.779 & 20.780 & 20.784 & 20.786 & 20.790 \\
\hline 9.4 & 20.971 & 20.970 & 20.968 & 20.966 & 20.964 & 20.962 & 20.961 & 20.961 & 20.969 & 20.974 & 20.983 \\
\hline 9.6 & 21.148 & 21.146 & 21.144 & 21.140 & 21.136 & 21.132 & 21.130 & 21.133 & 21.140 & 21.152 & 21.168 \\
\hline 9.8 & 21.310 & 21.307 & 21.303 & 21.297 & 21.291 & 21.285 & 21.279 & 21.281 & 21.289 & 21.314 & 21.339 \\
\hline 10.0 & 21.448 & 21.443 & 21.437 & 21.429 & 21.419 & 21.411 & 21.401 & 21.399 & 21.406 & 21.445 & 21.486 \\
\hline 10.2 & 21.553 & 21.547 & 21.539 & 21.529 & 21.515 & 21.502 & 21.489 & 21.481 & 21.500 & 21.536 & 21.599 \\
\hline 10.4 & 21.622 & 21.614 & 21.604 & 21.592 & 21.574 & 21.557 & 21.541 & 21.527 & 21.541 & 21.582 & 21.673 \\
\hline 10.6 & 21.655 & 21.646 & 21.633 & 21.620 & 21.600 & 21.579 & 21.560 & 21.542 & 21.547 & 21.584 & 21.697 \\
\hline 10.8 & 21.658 & 21.648 & 21.634 & 21.620 & 21.600 & 21.575 & 21.554 & 21.533 & 21.529 & 21.580 & 21.677 \\
\hline 11.0 & 21.642 & 21.629 & 21.616 & 21.600 & 21.581 & 21.555 & 21.530 & 21.509 & 21.496 & 21.533 & 21.624 \\
\hline
\end{tabular}

Table 14: $\log \sigma_{1}$ for ${ }^{56} \mathrm{Fe}$ at $B_{12}=100$

\begin{tabular}{|c|c|c|c|c|c|c|c|c|c|c|c|}
\hline $\log \rho$ & $\log T=-1.0$ & $\log T=-0.8$ & $\log T=-0.6$ & $\log T=-0.4$ & $\log T=-0.2$ & $\log T=0.0$ & $\log T=0.2$ & $\log T=0.4$ & $\log T=0.6$ & $\log T=0.8$ & $\log T=1.0$ \\
\hline 6.0 & 16.601 & 16.601 & 16.601 & 16.601 & 16.597 & 16.596 & 16.597 & 16.597 & 16.597 & 16.597 & 16.597 \\
\hline 6.2 & 16.801 & 16.801 & 16.801 & 16.801 & 16.799 & 16.796 & 16.797 & 16.797 & 16.797 & 16.797 & 16.797 \\
\hline 6.4 & 17.001 & 17.001 & 17.001 & 17.001 & 17.000 & 16.996 & 16.997 & 16.997 & 16.997 & 16.997 & 16.997 \\
\hline 6.6 & 17.201 & 17.201 & 17.201 & 17.201 & 17.201 & 17.196 & 17.197 & 17.197 & 17.197 & 17.197 & 17.197 \\
\hline 6.8 & 17.401 & 17.401 & 17.401 & 17.401 & 17.401 & 17.396 & 17.397 & 17.397 & 17.397 & 17.397 & 17.397 \\
\hline 7.0 & 17.601 & 17.601 & 17.601 & 17.601 & 17.601 & 17.596 & 17.597 & 17.597 & 17.597 & 17.597 & 17.597 \\
\hline 7.2 & 17.801 & 17.801 & 17.801 & 17.801 & 17.801 & 17.799 & 17.797 & 17.797 & 17.797 & 17.797 & 17.797 \\
\hline 7.4 & 18.001 & 18.001 & 18.001 & 18.001 & 18.001 & 18.001 & 17.997 & 17.997 & 17.997 & 17.997 & 17.997 \\
\hline 7.6 & 18.201 & 18.201 & 18.201 & 18.201 & 18.201 & 18.201 & 18.197 & 18.197 & 18.197 & 18.197 & 18.197 \\
\hline 7.8 & 18.401 & 18.401 & 18.401 & 18.401 & 18.401 & 18.401 & 18.398 & 18.397 & 18.397 & 18.397 & 18.397 \\
\hline 8.0 & 18.601 & 18.601 & 18.601 & 18.601 & 18.601 & 18.601 & 18.600 & 18.597 & 18.597 & 18.597 & 18.597 \\
\hline 8.2 & 18.801 & 18.801 & 18.801 & 18.801 & 18.801 & 18.801 & 18.801 & 18.797 & 18.797 & 18.797 & 18.797 \\
\hline 8.4 & 19.001 & 19.001 & 19.001 & 19.001 & 19.001 & 19.001 & 19.001 & 18.997 & 18.997 & 18.997 & 18.997 \\
\hline 8.6 & 19.201 & 19.201 & 19.201 & 19.201 & 19.201 & 19.201 & 19.201 & 19.200 & 19.197 & 19.197 & 19.197 \\
\hline 8.8 & 19.401 & 19.401 & 19.401 & 19.401 & 19.401 & 19.401 & 19.401 & 19.401 & 19.397 & 19.397 & 19.397 \\
\hline 9.0 & 19.601 & 19.601 & 19.601 & 19.601 & 19.601 & 19.601 & 19.601 & 19.601 & 19.597 & 19.597 & 19.597 \\
\hline 9.2 & 19.801 & 19.801 & 19.801 & 19.801 & 19.801 & 19.801 & 19.801 & 19.801 & 19.800 & 19.797 & 19.797 \\
\hline 9.4 & 20.001 & 20.001 & 20.001 & 20.001 & 20.001 & 20.001 & 20.001 & 20.001 & 20.001 & 19.996 & 19.997 \\
\hline 9.6 & 20.201 & 20.201 & 20.201 & 20.200 & 20.200 & 20.200 & 20.200 & 20.200 & 20.200 & 20.196 & 20.196 \\
\hline 9.8 & 20.400 & 20.400 & 20.400 & 20.400 & 20.400 & 20.400 & 20.400 & 20.400 & 20.400 & 20.399 & 20.396 \\
\hline 10.0 & 20.599 & 20.599 & 20.599 & 20.599 & 20.599 & 20.599 & 20.599 & 20.599 & 20.599 & 20.599 & 20.595 \\
\hline 10.2 & 20.798 & 20.798 & 20.798 & 20.797 & 20.797 & 20.797 & 20.797 & 20.796 & 20.797 & 20.797 & 20.794 \\
\hline 10.4 & 20.995 & 20.995 & 20.995 & 20.994 & 20.994 & 20.993 & 20.993 & 20.993 & 20.993 & 20.993 & 20.994 \\
\hline 10.6 & 21.190 & 21.190 & 21.190 & 21.189 & 21.188 & 21.187 & 21.187 & 21.186 & 21.185 & 21.186 & 21.189 \\
\hline 10.8 & 21.382 & 21.381 & 21.381 & 21.380 & 21.379 & 21.377 & 21.376 & 21.374 & 21.373 & 21.374 & 21.377 \\
\hline 11.0 & 21.558 & 21.566 & 21.565 & 21.564 & 21.562 & 21.559 & 21.557 & 21.554 & 21.551 & 21.553 & 21.556 \\
\hline
\end{tabular}

Table 15: $\log \sigma$ for density-dependent composition

\begin{tabular}{|c|c|c|c|c|c|c|c|c|c|c|c|}
\hline $\log \rho$ & $\log T=-1.0$ & $\log T=-0.8$ & $\log T=-0.6$ & $\log T=-0.4$ & $\log T=-0.2$ & $\log T=0.0$ & $\log T=0.2$ & $\log T=0.4$ & $\log T=0.6$ & $\log T=0.8$ & $\log T=1.0$ \\
\hline 6.0 & 20.144 & 20.260 & 20.376 & 20.506 & 20.628 & 20.762 & 20.902 & 21.048 & 21.200 & 21.357 & 21.522 \\
\hline 6.2 & 20.213 & 20.310 & 20.413 & 20.537 & 20.658 & 20.785 & 20.923 & 21.066 & 21.216 & 21.372 & 21.536 \\
\hline 6.4 & 20.293 & 20.368 & 20.456 & 20.570 & 20.689 & 20.809 & 20.944 & 21.085 & 21.233 & 21.388 & 21.550 \\
\hline 6.6 & 20.382 & 20.435 & 20.517 & 20.607 & 20.720 & 20.834 & 20.966 & 21.104 & 21.250 & 21.403 & 21.565 \\
\hline 6.8 & 20.477 & 20.510 & 20.572 & 20.647 & 20.753 & 20.861 & 20.989 & 21.125 & 21.268 & 21.420 & 21.580 \\
\hline 7.0 & 20.536 & 20.555 & 20.598 & 20.671 & 20.755 & 20.857 & 20.981 & 21.114 & 21.256 & 21.406 & 21.565 \\
\hline 7.2 & 20.633 & 20.641 & 20.666 & 20.721 & 20.792 & 20.893 & 21.006 & 21.136 & 21.276 & 21.424 & 21.581 \\
\hline 7.4 & 20.730 & 20.730 & 20.740 & 20.777 & 20.833 & 20.928 & 21.033 & 21.160 & 21.296 & 21.442 & 21.598 \\
\hline 7.6 & 20.823 & 20.817 & 20.819 & 20.839 & 20.893 & 20.964 & 21.062 & 21.184 & 21.317 & 21.461 & 21.615 \\
\hline 7.8 & 20.912 & 20.903 & 20.899 & 20.907 & 20.944 & 21.003 & 21.094 & 21.209 & 21.339 & 21.481 & 21.633 \\
\hline 8.0 & 20.998 & 20.987 & 20.980 & 20.979 & 21.001 & 21.044 & 21.132 & 21.236 & 21.363 & 21.501 & 21.652 \\
\hline 8.2 & 21.080 & 21.068 & 21.059 & 21.053 & 21.063 & 21.105 & 21.169 & 21.265 & 21.387 & 21.523 & 21.671 \\
\hline 8.4 & 21.160 & 21.147 & 21.136 & 21.128 & 21.129 & 21.157 & 21.208 & 21.296 & 21.412 & 21.545 & 21.691 \\
\hline 8.6 & 21.232 & 21.220 & 21.207 & 21.198 & 21.193 & 21.209 & 21.248 & 21.332 & 21.438 & 21.567 & 21.711 \\
\hline 8.8 & 21.307 & 21.296 & 21.283 & 21.273 & 21.264 & 21.270 & 21.308 & 21.370 & 21.466 & 21.592 & 21.733 \\
\hline 9.0 & 21.380 & 21.371 & 21.358 & 21.346 & 21.337 & 21.334 & 21.360 & 21.409 & 21.497 & 21.617 & 21.756 \\
\hline 9.2 & 21.311 & 21.304 & 21.297 & 21.288 & 21.282 & 21.284 & 21.302 & 21.353 & 21.428 & 21.540 & 21.679 \\
\hline 9.4 & 21.382 & 21.375 & 21.369 & 21.360 & 21.352 & 21.351 & 21.362 & 21.400 & 21.465 & 21.569 & 21.704 \\
\hline 9.6 & 21.478 & 21.472 & 21.465 & 21.455 & 21.446 & 21.442 & 21.445 & 21.472 & 21.539 & 21.621 & 21.751 \\
\hline 9.8 & 21.549 & 21.543 & 21.536 & 21.527 & 21.517 & 21.511 & 21.511 & 21.528 & 21.581 & 21.659 & 21.779 \\
\hline 10.0 & 21.619 & 21.614 & 21.606 & 21.599 & 21.589 & 21.580 & 21.579 & 21.588 & 21.628 & 21.698 & 21.809 \\
\hline 10.2 & 21.717 & 21.712 & 21.704 & 21.696 & 21.686 & 21.675 & 21.670 & 21.673 & 21.702 & 21.761 & 21.863 \\
\hline 10.4 & 21.787 & 21.782 & 21.776 & 21.768 & 21.758 & 21.747 & 21.741 & 21.739 & 21.758 & 21.817 & 21.903 \\
\hline 10.6 & 21.888 & 21.883 & 21.876 & 21.868 & 21.858 & 21.846 & 21.837 & 21.831 & 21.841 & 21.888 & 21.967 \\
\hline 10.8 & 21.976 & 21.970 & 21.964 & 21.955 & 21.946 & 21.933 & 21.922 & 21.915 & 21.917 & 21.952 & 22.021 \\
\hline 11.0 & - & 22.055 & 22.049 & 22.040 & 22.031 & 22.018 & 22.006 & 21.997 & 21.994 & 22.019 & 22.076 \\
\hline
\end{tabular}


Table 16: $\log \sigma_{0}$ for density-dependent composition at $B_{12}=1$

\begin{tabular}{|c|c|c|c|c|c|c|c|c|c|c|c|}
\hline $\log \rho$ & $\log T=-1.0$ & $\log T=-0.8$ & $\log T=-0.6$ & $\log T=-0.4$ & $\log T=-0.2$ & $\log T=0.0$ & $\log T=0.2$ & $\log T=0.4$ & $\log T=0.6$ & $\log T=0.8$ & $\log T=1.0$ \\
\hline 6.0 & 17.169 & 17.065 & 16.945 & 16.800 & 16.654 & 16.512 & 16.370 & 16.226 & 16.078 & 15.925 & 15.765 \\
\hline 6.2 & 17.472 & 17.395 & 17.294 & 17.161 & 17.023 & 16.885 & 16.748 & 16.607 & 16.460 & 16.309 & 16.151 \\
\hline 6.4 & 17.768 & 17.715 & 17.637 & 17.519 & 17.390 & 17.258 & 17.124 & 16.986 & 16.842 & 16.692 & 16.536 \\
\hline 6.6 & 18.057 & 18.026 & 17.957 & 17.872 & 17.754 & 17.628 & 17.499 & 17.364 & 17.223 & 17.075 & 16.920 \\
\hline 6.8 & 18.345 & 18.331 & 18.285 & 18.219 & 18.115 & 17.997 & 17.873 & 17.741 & 17.603 & 17.457 & 17.304 \\
\hline 7.0 & 18.650 & 18.645 & 18.617 & 18.555 & 18.480 & 18.370 & 18.252 & 18.124 & 17.989 & 17.845 & 17.694 \\
\hline 7.2 & 18.943 & 18.943 & 18.931 & 18.889 & 18.830 & 18.733 & 18.621 & 18.499 & 18.367 & 18.226 & 18.076 \\
\hline 7.4 & 19.238 & 19.242 & 19.240 & 19.216 & 19.174 & 19.090 & 18.986 & 18.870 & 18.743 & 18.605 & 18.458 \\
\hline 7.6 & 19.534 & 19.541 & 19.544 & 19.533 & 19.494 & 19.439 & 19.346 & 19.238 & 19.116 & 18.982 & 18.838 \\
\hline 7.8 & 19.829 & 19.837 & 19.843 & 19.841 & 19.817 & 19.777 & 19.697 & 19.599 & 19.485 & 19.357 & 19.217 \\
\hline 8.0 & 20.119 & 20.127 & 20.133 & 20.136 & 20.124 & 20.098 & 20.035 & 19.949 & 19.846 & 19.726 & 19.591 \\
\hline 8.2 & 20.397 & 20.404 & 20.409 & 20.413 & 20.408 & 20.386 & 20.350 & 20.281 & 20.193 & 20.085 & 19.960 \\
\hline 8.4 & 20.655 & 20.660 & 20.663 & 20.666 & 20.664 & 20.653 & 20.632 & 20.585 & 20.518 & 20.429 & 20.317 \\
\hline 8.6 & 20.871 & 20.872 & 20.873 & 20.873 & 20.872 & 20.867 & 20.858 & 20.835 & 20.791 & 20.725 & 20.633 \\
\hline 8.8 & 21.069 & 21.067 & 21.064 & 21.062 & 21.059 & 21.056 & 21.058 & 21.056 & 21.042 & 21.008 & 20.945 \\
\hline 9.0 & 21.232 & 21.228 & 21.222 & 21.216 & 21.211 & 21.208 & 21.214 & 21.226 & 21.240 & 21.243 & 21.217 \\
\hline 9.2 & 21.258 & 21.253 & 21.247 & 21.240 & 21.235 & 21.236 & 21.247 & 21.281 & 21.323 & 21.374 & 21.410 \\
\hline 9.4 & 21.352 & 21.346 & 21.341 & 21.333 & 21.326 & 21.325 & 21.333 & 21.363 & 21.412 & 21.482 & 21.553 \\
\hline 9.6 & 21.458 & 21.452 & 21.446 & 21.437 & 21.428 & 21.425 & 21.427 & 21.450 & 21.507 & 21.571 & 21.662 \\
\hline 9.8 & 21.537 & 21.532 & 21.525 & 21.517 & 21.507 & 21.501 & 21.501 & 21.517 & 21.566 & 21.635 & 21.735 \\
\hline 10.0 & 21.613 & 21.608 & 21.601 & 21.593 & 21.583 & 21.575 & 21.573 & 21.582 & 21.621 & 21.687 & 21.788 \\
\hline 10.2 & 21.713 & 21.708 & 21.700 & 21.693 & 21.682 & 21.672 & 21.667 & 21.669 & 21.697 & 21.754 & 21.851 \\
\hline 10.4 & 21.785 & 21.780 & 21.773 & 21.766 & 21.756 & 21.745 & 21.739 & 21.737 & 21.755 & 21.814 & 21.897 \\
\hline 10.6 & 21.887 & 21.882 & 21.874 & 21.867 & 21.857 & 21.844 & 21.835 & 21.830 & 21.839 & 21.886 & 21.964 \\
\hline 10.8 & 21.975 & 21.969 & 21.963 & 21.955 & 21.945 & 21.932 & 21.921 & 21.914 & 21.916 & 21.951 & 22.019 \\
\hline 11.0 & - & 22.055 & 22.048 & 22.040 & 22.031 & 22.018 & 22.005 & 21.996 & 21.993 & 22.018 & 22.075 \\
\hline
\end{tabular}

Table 17: $\log \sigma_{0}$ for density-dependent composition at $B_{12}=10$

\begin{tabular}{|c|c|c|c|c|c|c|c|c|c|c|c|}
\hline $\log \rho$ & $\log T=-1.0$ & $\log T=-0.8$ & $\log T=-0.6$ & $\log T=-0.4$ & $\log T=-0.2$ & $\log T=0.0$ & $\log T=0.2$ & $\log T=0.4$ & $\log T=0.6$ & $\log T=0.8$ & $\log T=1.0$ \\
\hline 6.0 & 15.171 & 15.068 & 14.948 & 14.801 & 14.654 & 14.512 & 14.370 & 14.226 & 14.078 & 13.925 & 13.765 \\
\hline 6.2 & 15.475 & 15.398 & 15.297 & 15.163 & 15.024 & 14.886 & 14.748 & 14.607 & 14.461 & 14.309 & 14.151 \\
\hline 6.4 & 15.771 & 15.718 & 15.641 & 15.521 & 15.392 & 15.259 & 15.124 & 14.986 & 14.842 & 14.692 & 14.536 \\
\hline 6.6 & 16.061 & 16.031 & 15.961 & 15.875 & 15.757 & 15.630 & 15.500 & 15.364 & 15.223 & 15.075 & 14.920 \\
\hline 6.8 & 16.350 & 16.336 & 16.290 & 16.224 & 16.118 & 15.999 & 15.874 & 15.742 & 15.603 & 15.457 & 15.304 \\
\hline 7.0 & 16.657 & 16.652 & 16.625 & 16.563 & 16.486 & 16.374 & 16.254 & 16.126 & 15.990 & 15.846 & 15.694 \\
\hline 7.2 & 16.952 & 16.954 & 16.942 & 16.901 & 16.840 & 16.740 & 16.625 & 16.501 & 16.368 & 16.227 & 16.077 \\
\hline 7.4 & 17.252 & 17.258 & 17.257 & 17.233 & 17.190 & 17.102 & 16.994 & 16.875 & 16.745 & 16.607 & 16.459 \\
\hline 7.6 & 17.557 & 17.565 & 17.570 & 17.560 & 17.519 & 17.461 & 17.361 & 17.248 & 17.122 & 16.986 & 16.840 \\
\hline 7.8 & 17.867 & 17.877 & 17.884 & 17.884 & 17.859 & 17.816 & 17.726 & 17.619 & 17.497 & 17.364 & 17.221 \\
\hline 8.0 & 18.180 & 18.192 & 18.201 & 18.207 & 18.195 & 18.166 & 18.089 & 17.988 & 17.871 & 17.741 & 17.601 \\
\hline 8.2 & 18.497 & 18.510 & 18.520 & 18.529 & 18.526 & 18.496 & 18.448 & 18.354 & 18.243 & 18.117 & 17.979 \\
\hline 8.4 & 18.817 & 18.829 & 18.840 & 18.850 & 18.855 & 18.838 & 18.802 & 18.718 & 18.613 & 18.492 & 18.357 \\
\hline 8.6 & 19.116 & 19.128 & 19.140 & 19.150 & 19.158 & 19.151 & 19.127 & 19.055 & 18.956 & 18.839 & 18.708 \\
\hline 8.8 & 19.438 & 19.449 & 19.461 & 19.472 & 19.482 & 19.482 & 19.456 & 19.411 & 19.320 & 19.210 & 19.082 \\
\hline 9.0 & 19.760 & 19.769 & 19.781 & 19.793 & 19.803 & 19.809 & 19.793 & 19.761 & 19.680 & 19.577 & 19.455 \\
\hline 9.2 & 20.169 & 20.175 & 20.181 & 20.189 & 20.195 & 20.194 & 20.185 & 20.150 & 20.094 & 19.999 & 19.883 \\
\hline 9.4 & 20.473 & 20.478 & 20.483 & 20.490 & 20.496 & 20.497 & 20.492 & 20.469 & 20.427 & 20.345 & 20.240 \\
\hline 9.6 & 20.721 & 20.726 & 20.730 & 20.736 & 20.742 & 20.745 & 20.743 & 20.729 & 20.691 & 20.632 & 20.540 \\
\hline 9.8 & 20.993 & 20.996 & 20.999 & 21.002 & 21.006 & 21.008 & 21.008 & 21.001 & 20.978 & 20.937 & 20.864 \\
\hline 10.0 & 21.234 & 21.235 & 21.236 & 21.237 & 21.238 & 21.239 & 21.238 & 21.235 & 21.225 & 21.203 & 21.156 \\
\hline 10.2 & 21.425 & 21.424 & 21.424 & 21.424 & 21.423 & 21.422 & 21.420 & 21.418 & 21.415 & 21.407 & 21.383 \\
\hline 10.4 & 21.603 & 21.601 & 21.599 & 21.597 & 21.593 & 21.589 & 21.586 & 21.583 & 21.585 & 21.594 & 21.596 \\
\hline 10.6 & 21.754 & 21.751 & 21.748 & 21.744 & 21.739 & 21.732 & 21.727 & 21.723 & 21.724 & 21.740 & 21.758 \\
\hline 10.8 & 21.887 & 21.884 & 21.880 & 21.874 & 21.868 & 21.859 & 21.851 & 21.845 & 21.845 & 21.863 & 21.893 \\
\hline 11.0 & - & 21.998 & 21.993 & 21.986 & 21.979 & 21.969 & 21.959 & 21.951 & 21.947 & 21.963 & 21.997 \\
\hline
\end{tabular}

Table 18: $\log \sigma_{0}$ for density-dependent composition at $B_{12}=100$

\begin{tabular}{|c|c|c|c|c|c|c|c|c|c|c|c|}
\hline $\log \rho$ & $\log T=-1.0$ & $\log T=-0.8$ & $\log T=-0.6$ & $\log T=-0.4$ & $\log T=-0.2$ & $\log T=0.0$ & $\log T=0.2$ & $\log T=0.4$ & $\log T=0.6$ & $\log T=0.8$ & $\log T=1.0$ \\
\hline 6.0 & 13.171 & 13.068 & 12.948 & 12.801 & 12.654 & 12.512 & 12.370 & 12.226 & 12.078 & 11.925 & 11.765 \\
\hline 6.2 & 13.475 & 13.398 & 13.297 & 13.163 & 13.024 & 12.886 & 12.748 & 12.607 & 12.461 & 12.309 & 12.151 \\
\hline 6.4 & 13.771 & 13.718 & 13.641 & 13.521 & 13.392 & 13.259 & 13.124 & 12.986 & 12.842 & 12.692 & 12.536 \\
\hline 6.6 & 14.061 & 14.031 & 13.961 & 13.875 & 13.757 & 13.630 & 13.500 & 13.364 & 13.223 & 13.075 & 12.920 \\
\hline 6.8 & 14.350 & 14.336 & 14.290 & 14.224 & 14.118 & 13.999 & 13.874 & 13.742 & 13.603 & 13.457 & 13.304 \\
\hline 7.0 & 14.657 & 14.652 & 14.625 & 14.563 & 14.486 & 14.374 & 14.254 & 14.126 & 13.990 & 13.846 & 13.694 \\
\hline 7.2 & 14.952 & 14.954 & 14.942 & 14.901 & 14.840 & 14.740 & 14.625 & 14.501 & 14.368 & 14.227 & 14.077 \\
\hline 7.4 & 15.252 & 15.258 & 15.257 & 15.233 & 15.190 & 15.102 & 14.994 & 14.875 & 14.745 & 14.607 & 14.459 \\
\hline 7.6 & 15.557 & 15.565 & 15.570 & 15.560 & 15.520 & 15.461 & 15.361 & 15.248 & 15.122 & 14.986 & 14.840 \\
\hline 7.8 & 15.867 & 15.877 & 15.885 & 15.885 & 15.860 & 15.816 & 15.727 & 15.619 & 15.497 & 15.364 & 15.221 \\
\hline 8.0 & 16.181 & 16.193 & 16.201 & 16.208 & 16.196 & 16.167 & 16.090 & 15.988 & 15.871 & 15.742 & 15.601 \\
\hline 8.2 & 16.498 & 16.511 & 16.521 & 16.530 & 16.528 & 16.498 & 16.449 & 16.355 & 16.244 & 16.118 & 15.980 \\
\hline 8.4 & 16.819 & 16.831 & 16.843 & 16.852 & 16.857 & 16.840 & 16.804 & 16.720 & 16.615 & 16.493 & 16.358 \\
\hline 8.6 & 17.119 & 17.131 & 17.144 & 17.154 & 17.163 & 17.155 & 17.132 & 17.058 & 16.958 & 16.841 & 16.708 \\
\hline 8.8 & 17.444 & 17.455 & 17.468 & 17.479 & 17.489 & 17.490 & 17.463 & 17.418 & 17.325 & 17.213 & 17.084 \\
\hline 9.0 & 17.771 & 17.780 & 17.793 & 17.805 & 17.816 & 17.822 & 17.807 & 17.773 & 17.690 & 17.584 & 17.459 \\
\hline 9.2 & 18.201 & 18.208 & 18.215 & 18.225 & 18.232 & 18.232 & 18.222 & 18.185 & 18.124 & 18.020 & 17.897 \\
\hline 9.4 & 18.530 & 18.536 & 18.543 & 18.552 & 18.561 & 18.563 & 18.559 & 18.532 & 18.484 & 18.387 & 18.269 \\
\hline 9.6 & 18.804 & 18.811 & 18.817 & 18.827 & 18.837 & 18.842 & 18.843 & 18.826 & 18.775 & 18.701 & 18.589 \\
\hline 9.8 & 19.133 & 19.139 & 19.146 & 19.154 & 19.164 & 19.172 & 19.174 & 19.165 & 19.127 & 19.064 & 18.957 \\
\hline 10.0 & 19.461 & 19.467 & 19.474 & 19.481 & 19.491 & 19.500 & 19.504 & 19.500 & 19.473 & 19.423 & 19.322 \\
\hline 10.2 & 19.730 & 19.735 & 19.743 & 19.750 & 19.761 & 19.771 & 19.777 & 19.779 & 19.762 & 19.723 & 19.631 \\
\hline 10.4 & 20.056 & 20.061 & 20.067 & 20.074 & 20.084 & 20.095 & 20.102 & 20.106 & 20.097 & 20.055 & 19.989 \\
\hline 10.6 & 20.317 & 20.322 & 20.328 & 20.336 & 20.345 & 20.357 & 20.366 & 20.373 & 20.370 & 20.339 & 20.284 \\
\hline 10.8 & 20.604 & 20.609 & 20.615 & 20.622 & 20.631 & 20.642 & 20.653 & 20.661 & 20.663 & 20.642 & 20.598 \\
\hline 11.0 & - & 20.872 & 20.878 & 20.885 & 20.893 & 20.904 & 20.915 & 20.923 & 20.928 & 20.915 & 20.882 \\
\hline
\end{tabular}


Table 19: $\log \sigma_{1}$ for density-dependent composition at $B_{12}=1$

\begin{tabular}{|c|c|c|c|c|c|c|c|c|c|c|c|}
\hline $\log \rho$ & $\log T=-1.0$ & $\log T=-0.8$ & $\log T=-0.6$ & $\log T=-0.4$ & $\log T=-0.2$ & $\log T=0.0$ & $\log T=0.2$ & $\log T=0.4$ & $\log T=0.6$ & $\log T=0.8$ & $\log T=1.0$ \\
\hline 6.0 & 18.600 & 18.601 & 18.601 & 18.601 & 18.597 & 18.596 & 18.597 & 18.597 & 18.597 & 18.597 & 18.597 \\
\hline 6.2 & 18.800 & 18.800 & 18.801 & 18.801 & 18.798 & 18.796 & 18.797 & 18.797 & 18.797 & 18.797 & 18.797 \\
\hline 6.4 & 18.999 & 19.000 & 19.000 & 19.000 & 18.999 & 18.996 & 18.996 & 18.997 & 18.997 & 18.997 & 18.997 \\
\hline 6.6 & 19.199 & 19.199 & 19.199 & 19.200 & 19.200 & 19.196 & 19.196 & 19.197 & 19.197 & 19.197 & 19.197 \\
\hline 6.8 & 19.398 & 19.398 & 19.398 & 19.399 & 19.399 & 19.396 & 19.396 & 19.396 & 19.397 & 19.397 & 19.397 \\
\hline 7.0 & 19.583 & 19.583 & 19.583 & 19.585 & 19.586 & 19.582 & 19.583 & 19.584 & 19.584 & 19.584 & 19.585 \\
\hline 7.2 & 19.780 & 19.780 & 19.780 & 19.781 & 19.783 & 19.783 & 19.782 & 19.783 & 19.784 & 19.784 & 19.784 \\
\hline 7.4 & 19.975 & 19.974 & 19.974 & 19.975 & 19.977 & 19.980 & 19.979 & 19.982 & 19.983 & 19.984 & 19.984 \\
\hline 7.6 & 20.166 & 20.165 & 20.164 & 20.165 & 20.168 & 20.173 & 20.174 & 20.178 & 20.181 & 20.183 & 20.184 \\
\hline 7.8 & 20.352 & 20.350 & 20.348 & 20.348 & 20.351 & 20.357 & 20.363 & 20.370 & 20.376 & 20.380 & 20.382 \\
\hline 8.0 & 20.527 & 20.524 & 20.522 & 20.520 & 20.522 & 20.529 & 20.544 & 20.555 & 20.567 & 20.574 & 20.579 \\
\hline 8.2 & 20.688 & 20.683 & 20.678 & 20.674 & 20.675 & 20.688 & 20.705 & 20.726 & 20.747 & 20.762 & 20.772 \\
\hline 8.4 & 20.826 & 20.818 & 20.810 & 20.804 & 20.801 & 20.814 & 20.837 & 20.873 & 20.910 & 20.938 & 20.958 \\
\hline 8.6 & 20.927 & 20.916 & 20.905 & 20.896 & 20.889 & 20.899 & 20.925 & 20.981 & 21.035 & 21.083 & 21.118 \\
\hline 8.8 & 21.000 & 20.987 & 20.972 & 20.960 & 20.948 & 20.953 & 20.993 & 21.052 & 21.130 & 21.208 & 21.268 \\
\hline 9.0 & 21.037 & 21.023 & 21.004 & 20.987 & 20.973 & 20.969 & 21.005 & 21.071 & 21.174 & 21.287 & 21.383 \\
\hline 9.2 & 20.813 & 20.801 & 20.788 & 20.772 & 20.762 & 20.768 & 20.803 & 20.897 & 21.021 & 21.185 & 21.349 \\
\hline 9.4 & 20.778 & 20.765 & 20.753 & 20.736 & 20.722 & 20.723 & 20.746 & 20.825 & 20.952 & 21.132 & 21.335 \\
\hline 9.6 & 20.795 & 20.782 & 20.769 & 20.751 & 20.733 & 20.727 & 20.737 & 20.797 & 20.933 & 21.092 & 21.316 \\
\hline 9.8 & 20.744 & 20.733 & 20.719 & 20.703 & 20.683 & 20.672 & 20.675 & 20.716 & 20.832 & 20.995 & 21.225 \\
\hline 10.0 & 20.690 & 20.680 & 20.665 & 20.651 & 20.631 & 20.615 & 20.614 & 20.638 & 20.731 & 20.884 & 21.110 \\
\hline 10.2 & 20.704 & 20.693 & 20.679 & 20.663 & 20.642 & 20.622 & 20.614 & 20.623 & 20.692 & 20.827 & 21.043 \\
\hline 10.4 & 20.647 & 20.637 & 20.623 & 20.608 & 20.589 & 20.567 & 20.555 & 20.556 & 20.603 & 20.738 & 20.927 \\
\hline 10.6 & 20.667 & 20.656 & 20.642 & 20.626 & 20.606 & 20.582 & 20.565 & 20.557 & 20.584 & 20.695 & 20.873 \\
\hline 10.8 & 20.652 & 20.641 & 20.627 & 20.611 & 20.592 & 20.566 & 20.545 & 20.532 & 20.543 & 20.629 & 20.789 \\
\hline 11.0 & - & 20.632 & 20.619 & 20.602 & 20.583 & 20.557 & 20.533 & 20.516 & 20.515 & 20.579 & 20.717 \\
\hline
\end{tabular}

Table 20: $\log \sigma_{1}$ for density-dependent composition at $B_{12}=10$

\begin{tabular}{|c|c|c|c|c|c|c|c|c|c|c|c|}
\hline $\log \rho$ & $\log T=-1.0$ & $\log T=-0.8$ & $\log T=-0.6$ & $\log T=-0.4$ & $\log T=-0.2$ & $\log T=0.0$ & $\log T=0.2$ & $\log T=0.4$ & $\log T=0.6$ & $\log T=0.8$ & $\log T=1.0$ \\
\hline 6.0 & 17.601 & 17.601 & 17.601 & 17.601 & 17.597 & 17.596 & 17.597 & 17.597 & 17.597 & 17.597 & 17.597 \\
\hline 6.2 & 17.801 & 17.801 & 17.801 & 17.801 & 17.799 & 17.796 & 17.797 & 17.797 & 17.797 & 17.797 & 17.797 \\
\hline 6.4 & 18.001 & 18.001 & 18.001 & 18.001 & 18.000 & 17.996 & 17.997 & 17.997 & 17.997 & 17.997 & 17.997 \\
\hline 6.6 & 18.201 & 18.201 & 18.201 & 18.201 & 18.201 & 18.196 & 18.197 & 18.197 & 18.197 & 18.197 & 18.197 \\
\hline 6.8 & 18.401 & 18.401 & 18.401 & 18.401 & 18.401 & 18.396 & 18.397 & 18.397 & 18.397 & 18.397 & 18.397 \\
\hline 7.0 & 18.589 & 18.589 & 18.589 & 18.589 & 18.589 & 18.584 & 18.585 & 18.585 & 18.585 & 18.585 & 18.585 \\
\hline 7.2 & 18.789 & 18.789 & 18.789 & 18.789 & 18.789 & 18.787 & 18.785 & 18.785 & 18.785 & 18.785 & 18.785 \\
\hline 7.4 & 18.989 & 18.989 & 18.989 & 18.989 & 18.989 & 18.988 & 18.984 & 18.985 & 18.985 & 18.985 & 18.985 \\
\hline 7.6 & 19.189 & 19.189 & 19.189 & 19.189 & 19.189 & 19.189 & 19.184 & 19.185 & 19.185 & 19.185 & 19.185 \\
\hline 7.8 & 19.389 & 19.389 & 19.389 & 19.389 & 19.389 & 19.389 & 19.385 & 19.384 & 19.385 & 19.385 & 19.385 \\
\hline 8.0 & 19.588 & 19.588 & 19.588 & 19.588 & 19.588 & 19.589 & 19.588 & 19.584 & 19.584 & 19.585 & 19.585 \\
\hline 8.2 & 19.788 & 19.788 & 19.788 & 19.788 & 19.788 & 19.788 & 19.788 & 19.784 & 19.784 & 19.784 & 19.785 \\
\hline 8.4 & 19.987 & 19.987 & 19.987 & 19.987 & 19.987 & 19.987 & 19.987 & 19.983 & 19.984 & 19.984 & 19.984 \\
\hline 8.6 & 20.172 & 20.172 & 20.172 & 20.171 & 20.171 & 20.171 & 20.172 & 20.171 & 20.169 & 20.170 & 20.170 \\
\hline 8.8 & 20.369 & 20.369 & 20.369 & 20.368 & 20.368 & 20.368 & 20.369 & 20.369 & 20.367 & 20.368 & 20.369 \\
\hline 9.0 & 20.565 & 20.564 & 20.564 & 20.563 & 20.562 & 20.562 & 20.563 & 20.564 & 20.563 & 20.566 & 20.568 \\
\hline 9.2 & 20.724 & 20.723 & 20.722 & 20.720 & 20.719 & 20.719 & 20.720 & 20.725 & 20.730 & 20.735 & 20.742 \\
\hline 9.4 & 20.899 & 20.897 & 20.896 & 20.893 & 20.891 & 20.890 & 20.891 & 20.897 & 20.907 & 20.918 & 20.931 \\
\hline 9.6 & 21.058 & 21.056 & 21.053 & 21.050 & 21.046 & 21.044 & 21.043 & 21.049 & 21.066 & 21.079 & 21.100 \\
\hline 9.8 & 21.200 & 21.197 & 21.193 & 21.188 & 21.181 & 21.177 & 21.175 & 21.181 & 21.204 & 21.231 & 21.264 \\
\hline 10.0 & 21.311 & 21.307 & 21.300 & 21.294 & 21.285 & 21.277 & 21.274 & 21.279 & 21.306 & 21.347 & 21.401 \\
\hline 10.2 & 21.416 & 21.410 & 21.403 & 21.394 & 21.383 & 21.371 & 21.365 & 21.365 & 21.391 & 21.438 & 21.511 \\
\hline 10.4 & 21.465 & 21.458 & 21.449 & 21.439 & 21.426 & 21.410 & 21.401 & 21.398 & 21.421 & 21.491 & 21.578 \\
\hline 10.6 & 21.534 & 21.526 & 21.515 & 21.504 & 21.488 & 21.470 & 21.456 & 21.447 & 21.462 & 21.530 & 21.630 \\
\hline 10.8 & 21.564 & 21.555 & 21.544 & 21.531 & 21.514 & 21.493 & 21.475 & 21.462 & 21.468 & 21.530 & 21.637 \\
\hline 11.0 & - & 21.575 & 21.563 & 21.548 & 21.532 & 21.508 & 21.486 & 21.471 & 21.468 & 21.518 & 21.623 \\
\hline
\end{tabular}

Table 21: $\log \sigma_{1}$ for density-dependent composition at $B_{12}=100$

\begin{tabular}{|c|c|c|c|c|c|c|c|c|c|c|c|}
\hline $\log \rho$ & $\log T=-1.0$ & $\log T=-0.8$ & $\log T=-0.6$ & $\log T=-0.4$ & $\log T=-0.2$ & $\log T=0.0$ & $\log T=0.2$ & $\log T=0.4$ & $\log T=0.6$ & $\log T=0.8$ & $\log T=1.0$ \\
\hline 6.0 & 16.601 & 16.601 & 16.601 & 16.601 & 16.597 & 16.596 & 16.597 & 16.597 & 16.597 & 16.597 & 16.597 \\
\hline 6.2 & 16.801 & 16.801 & 16.801 & 16.801 & 16.799 & 16.796 & 16.797 & 16.797 & 16.797 & 16.797 & 16.797 \\
\hline 6.4 & 17.001 & 17.001 & 17.001 & 17.001 & 17.000 & 16.996 & 16.997 & 16.997 & 16.997 & 16.997 & 16.997 \\
\hline 6.6 & 17.201 & 17.201 & 17.201 & 17.201 & 17.201 & 17.196 & 17.197 & 17.197 & 17.197 & 17.197 & 17.197 \\
\hline 6.8 & 17.401 & 17.401 & 17.401 & 17.401 & 17.401 & 17.396 & 17.397 & 17.397 & 17.397 & 17.397 & 17.397 \\
\hline 7.0 & 17.589 & 17.589 & 17.589 & 17.589 & 17.589 & 17.584 & 17.585 & 17.585 & 17.585 & 17.585 & 17.585 \\
\hline 7.2 & 17.789 & 17.789 & 17.789 & 17.789 & 17.789 & 17.787 & 17.785 & 17.785 & 17.785 & 17.785 & 17.785 \\
\hline 7.4 & 17.989 & 17.989 & 17.989 & 17.989 & 17.989 & 17.989 & 17.985 & 17.985 & 17.985 & 17.985 & 17.985 \\
\hline 7.6 & 18.189 & 18.189 & 18.189 & 18.189 & 18.189 & 18.189 & 18.185 & 18.185 & 18.185 & 18.185 & 18.185 \\
\hline 7.8 & 18.389 & 18.389 & 18.389 & 18.389 & 18.389 & 18.389 & 18.386 & 18.385 & 18.385 & 18.385 & 18.385 \\
\hline 8.0 & 18.589 & 18.589 & 18.589 & 18.589 & 18.589 & 18.589 & 18.588 & 18.585 & 18.585 & 18.585 & 18.585 \\
\hline 8.2 & 18.789 & 18.789 & 18.789 & 18.789 & 18.789 & 18.789 & 18.789 & 18.785 & 18.785 & 18.785 & 18.785 \\
\hline 8.4 & 18.989 & 18.989 & 18.989 & 18.989 & 18.989 & 18.989 & 18.989 & 18.985 & 18.985 & 18.985 & 18.985 \\
\hline 8.6 & 19.175 & 19.175 & 19.175 & 19.175 & 19.175 & 19.175 & 19.175 & 19.174 & 19.171 & 19.171 & 19.171 \\
\hline 8.8 & 19.375 & 19.375 & 19.375 & 19.375 & 19.375 & 19.375 & 19.375 & 19.375 & 19.371 & 19.371 & 19.371 \\
\hline 9.0 & 19.575 & 19.575 & 19.575 & 19.575 & 19.575 & 19.575 & 19.575 & 19.575 & 19.571 & 19.571 & 19.571 \\
\hline 9.2 & 19.756 & 19.756 & 19.756 & 19.756 & 19.756 & 19.756 & 19.756 & 19.756 & 19.754 & 19.752 & 19.752 \\
\hline 9.4 & 19.956 & 19.956 & 19.956 & 19.955 & 19.956 & 19.956 & 19.956 & 19.956 & 19.955 & 19.951 & 19.951 \\
\hline 9.6 & 20.141 & 20.141 & 20.141 & 20.141 & 20.141 & 20.141 & 20.141 & 20.141 & 20.141 & 20.136 & 20.137 \\
\hline 9.8 & 20.340 & 20.340 & 20.340 & 20.340 & 20.340 & 20.340 & 20.340 & 20.340 & 20.340 & 20.338 & 20.336 \\
\hline 10.0 & 20.539 & 20.538 & 20.538 & 20.538 & 20.538 & 20.538 & 20.538 & 20.538 & 20.538 & 20.538 & 20.535 \\
\hline 10.2 & 20.721 & 20.721 & 20.721 & 20.721 & 20.720 & 20.720 & 20.720 & 20.720 & 20.720 & 20.721 & 20.718 \\
\hline 10.4 & 20.918 & 20.917 & 20.917 & 20.917 & 20.916 & 20.916 & 20.916 & 20.915 & 20.916 & 20.917 & 20.917 \\
\hline 10.6 & 21.097 & 21.096 & 21.096 & 21.095 & 21.095 & 21.094 & 21.093 & 21.093 & 21.093 & 21.095 & 21.097 \\
\hline 10.8 & 21.280 & 21.280 & 21.279 & 21.279 & 21.278 & 21.276 & 21.275 & 21.274 & 21.273 & 21.275 & 21.279 \\
\hline 11.0 & - & 21.449 & 21.448 & 21.447 & 21.446 & 21.444 & 21.442 & 21.440 & 21.438 & 21.440 & 21.444 \\
\hline
\end{tabular}

